\title{
L'HOMME L'Homme
}

Revue française d'anthropologie

185-186 | 2008

L'anthropologue et le contemporain : autour de Marc Augé

\section{L'Afrique en tête}

Jean Jamin

\section{OpenEdition}

Journals

Édition électronique

URL : http://journals.openedition.org/lhomme/24210

DOI : 10.4000//homme.24210

ISSN : 1953-8103

Éditeur

Éditions de l'EHESS

\section{Édition imprimée}

Date de publication : 1 janvier 2008

Pagination : 401-439

ISSN : 0439-4216

\section{Référence électronique}

Jean Jamin, «L'Afrique en tête », L'Homme [En ligne], 185-186 | 2008, mis en ligne le 01 janvier 2010, consulté le 19 avril 2019. URL : http://journals.openedition.org//homme/24210 ; DOI : 10.4000/ Ihomme.24210

\section{(C) École des hautes études en sciences sociales}




\title{
L'Afrique en tête
}

\begin{abstract}
Jean Jamin
Quand l'homme a voulu imiter la marche, il a créé la roue qui ne ressemble pas à une jambe. Il a fait ainsi du surréalisme sans le savoir. Guillaume Apollinaire, Les Mamelles de Tirésias.

À l'origine n'est pas le mot, mais la phrase, une modulation. Écoutez le chant des oiseaux. Blaise Cendrars, Blaise Cendrars vous parle...
\end{abstract}

L - E TITRE QUE JE PROPOSE ici en hommage à Marc Augé m'a été inspiré par une de ces phrases en boulet de canon qu'il nous arrive de prononcer mentalement entre le sommeil et l'éveil, phrases auxquelles Michel Leiris accordait toute son attention, accrochait sa mémoire, exerçait son flair de limier du langage, et sur lesquelles, prenant soin, sitôt survenues, de les recopier dans les pages de son agenda, de son journal ou sur de simples fiches, il aima gloser au fil des volumes de son autobiographie, mais à la manière d'un géomancien traçant de nombreuses et complexes figures stylistiques (incises, appositions, enchâssements, parataxes, périodes longues) comme pour mieux en extirper la valeur escomptée d'oracles.

À l'aube du 30 octobre 1972, il note la phrase qui vient de lui traverser l'esprit (Leiris 2003: 1253). C'est presque un alexandrin, version en quelque sorte lettrée de la sensation proustienne : "Ici fruit à la tête se dit: là on s'enlise ". Il y voit - je passe sur la longue exégèse qu'il en fait dans Frêle bruit (Ibid. : 1043-1053) - une sentence qui aurait signifié sa vie de poète et de savant sans oublier, en dépit des antinomies auxquelles peut donner lieu une telle imbrication, celle de ses engagements (conjugaux, amicaux, moraux, idéologiques, politiques). Par l'opposition entre le proche

Une première version de cet article réalisée sous forme de diaporama avec le logiciel Power Point qui permit d'associer textes, images et sons, a été présentée fin mai 2007 dans deux séminaires de l'École des hautes études en sciences sociales, puis à la journée d'étude L'Afrique, miroir du contemporain, organisée le mardi 19 juin 2007 par l'Institut interdisciplinaire d'anthropologie du contemporain (IIAC) au sein de la même institution. Je remercie François Flahault, Jean-Paul Colleyn, Jean-Pierre Dozon et Véronique Nahoum-Grappe de leurs observations et commentaires après la projection du diaporama, ainsi que Marc Chemillier et Bernard Lortat-Jacob de leurs critiques et suggestions au moment de la rédaction proprement dite de l'article. 
et le lointain, le dedans et le dehors, le convexe et le concave qu'introduisent et traduisent, même phonétiquement, les adverbes de lieu scandant l'énoncé, la sentence jugée par lui "cruciale » aurait cristallisé sa vocation d'autobiographe (ici) et sa carrière d'ethnographe (là) sauf que le bourgeonnement espéré de l'une (ici fruit à la tête) pouvait se retourner comme un gant (le pronominal se dire) et se corrompre dans l'envasement redouté de l'autre (là on s'enlise).

L'Afrique fantôme, publiée en 1934 (Leiris 1996) au lendemain de la célèbre mission ethnographique et linguistique Dakar-Djibouti (19311933), relate ce balancement périlleux entre les fragiles protubérances du "fruit à la tête " - la construction de soi par la découverte de l'autre - et les «sables mouvants» de la réalité coloniale où l'on risquait de perdre pied, tête y comprise.

Au lieu de le parler, c'était le langage qui se mettait soudain, dans un état de demi-conscience, à parler en soi (et de soi).

\section{Appareillage}

Vers le début de La Traversée du Luxembourg, Marc Augé (1985: 22) dresse un constat analogue, s'interrogeant sur le surgissement en lui au petit matin, dans un Paris estival déjà dépeuplé, d'un vrai alexandrin cette fois, issu tout droit et tout net, c'est-à-dire sans médiation, d'une tragédie de Racine: "Dans l'Orient désert, quel devint mon ennui!", comme si la «tristesse majestueuse » exprimée par Antiochus, prince d'Orient revenu à Rome par amour pour une Bérénice aux yeux désespérément distraits, venait subitement lui rappeler la double étrangeté, voire la mélancolie que ressent l'ethnologue de n'être jamais chez soi nulle part, ni sur le terrain où il lui arrive pourtant de séjourner longuement et familièrement, ni à son retour, parmi ses proches et ses collègues, dans son appartement, derrière son bureau, face à ses livres et ses notes. Claude Lévi-Strauss (1955 : 1217) avait déjà mis l'accent sur le déracinement chronique auquel l'ethnographe est voué professionnellement, proposant même une conception quasi dantesque de ses pérégrinations et de son expérience que j'ai considérée un moment comme désabusée et terriblement déprimante (Jamin 1982: 27 sq.), mais une conception par rapport à laquelle Marc Augé gardera toujours une distance critique, lui qui reconnaît volontiers ne pas haïr les voyages (Augé 1985: 24) ni craindre le dépaysement qu'ils provoquent et sur quoi justement se fondent non seulement le «récit de vie » de l'ethnologue mais une large part de la vérité objective des autres qu'il est censé observer, transcrire, décrire, analyser (Augé 2000 : 73 sq.). Si l'ethnologue se déplace dans plusieurs mondes et, ce faisant, se désubjectivise, se déréalise, ceux qu’il 
étudie le font tout autant, à leur façon, que ce soit par leur commerce avec les esprits (chamanisme, transe, possession) ou par leur exploration concrète, parfois méthodique, des côtés obscurs de la personne et de la personnalité (rêve, hallucination, mauvais œil). L'altérité fait bien partie de l'identité.

Aussi peut-on voir autre chose qu'une simple nostalgie dans le vers de Bérénice, dont Marc Augé perçoit d'ailleurs le décalage, si ce n'est l'ironie de son apparition. Ce vers est venu distraitement se greffer sur le refrain d'une publicité entendue à la radio quelques minutes plus tôt vantant la "fraîcheur de vivre" que procurerait une marque célèbre de chewinggum; puis il s'est emparé de lui au point d'aiguiller sa propre vision des choses, fussent-elles les plus ordinaires, et d'empreindre son propre phrasé : "Ce que c'est que de citer Racine », observe-t-il (Augé 1985 : 22), "je ne peux plus penser qu'en vers de douze pieds».

Au-delà du sens explicite de l'alexandrin, et quelles que soient les raisons qui ont conduit le héros racinien à errer pendant cinq ans en Palestine pour n'y trouver en fin de compte que rides et ruines, cette réminiscence se rapproche formellement de la phrase de Leiris et agirait comme une prémonition : elle annonce ce à quoi Marc Augé va désormais consacrer quelquesunes de ses observations, analyses et réflexions. Non seulement prendre à bras le corps aussi bien que par la pointe de la plume le tangage existentiel de l'ethnologue, qui n'est, somme toute, qu'un fort grossissement de la situation ordinaire de la condition sociale (in Bessis 2004 : 17), mais - ce qui en est le corollaire - s'interroger sur la construction intellectuelle de ses objets, qu'ils soient lointains ou proches, où pèsent (et sans doute ont toujours pesé) de grandes parts d'ombre et d'incertitude sur l'identité de soi et d'aussi grandes difficultés à établir et maintenir des relations avec autrui comme à entretenir des rapports étroits et efficients avec la nature ou avec les dieux. Autant de problèmes, voire de casse-têtes symboliques, cognitifs et techniques auxquels toute société, toute humanité, à quelque échelle et en quelque lieu que ce soit, se trouve confrontée mais que le processus désormais identifié par le terme de mondialisation - Marc Augé (2003b : 134) préfere parler d'urbanisation du monde - aurait multipliés, redessinant le puzzle social et culturel de la planète au risque de ne plus savoir en assembler les morceaux, ni même de reconnaître les arêtes qui devraient leur permettre de se joindre.

Les non-lieux que Marc Augé (1992) examinera et conceptualisera plus tard en sont comme l'architectonie: espaces de bifurcation, d'entrecroisement, de superposition de voies, de lignes et de signes, néanmoins strictement codifiés, qui semblent produire des identités et des relations 
sociales en stand-by ${ }^{1}$, c'est-à-dire temporaires et variables, dans la mesure où, pour reprendre quelques-uns de ses objets de prédilection - grandes surfaces, échangeurs autoroutiers, gares, aéroports, parcs de loisir, stations de métro, fussent-elles, par leurs noms, évocatrices d'histoires et de souvenirs -, on n'y est plus que chalands, consommateurs, visiteurs, spectateurs, usagers, ou encore et surtout passagers de convois soit dits en partance, soit dits en provenance, soit encore dits en transit ou en suspens, autrement dit en grève (c'est l'inénarrable message : "Suite à un mouvement social...»). - Des passagers souvent en quête, en attente ou en rêve d'un Orient dont les déserts déjà inscrits dans l'architecture dédaléenne de ces non-lieux viendraient conjurer l'ennui auquel, tel Antiochus, ils pourraient craindre de succomber une fois rendus sur place pour en fouler le sable et les pierres.

Même si les non-lieux brouillent les repères, égarent, inquiètent par leur surdimensionnement, leur surpopulation, leur surcharge signalétique - il y a toujours de l'excès dans les déserts, et l'on sait que la sensation de solitude peut être aussi vive au milieu d'une foule qu'au sommet des dunes ou au pied des ruines -, il arrive, remarque Marc Augé (in Bessis 2004 : 54), qu'on y éprouve une sensation d'apesanteur, une sorte de pause relationnelle comme si on entrait soudain dans un « repos identitaire ». Mais c'est d'un repos paradoxal qu'il s'agit en ce cas, comme on peut le dire de la phase de sommeil où se déploie une importante activité cérébrale qui, ce faisant, ouvre la porte aux rêves. Car, aux entrées et sorties de ces nonlieux, s'exerce une intense, sourcilleuse et parfois déroutante activité identificatoire, que ce soit sous forme numérique (caméras de vidéosurveillance), chiffrée (codes bancaires), perforée (compostage), optique (codes barres), ou à présent biométrique (passeports), et bientôt génétique... Bref, une activité qui s'apparente - ceci est à peine une métaphore - à une véritable fouille au corps. Le repos n'est donc lui aussi que passager, quand bien même voudrait-on prolonger cet entre-deux où l'identité individuelle et les relations sociales sont en suspens, en somme en vacance, quoique - parce que - mécaniquement gérées : d'abord on ne connaît ni ne choisit son voisin de cabine ou de compartiment.

Sans doute est-ce là une des premières "biffures" symboliques auxquelles Marc Augé, dans le sillage de Michel Leiris², accordera de l'importance dans ses réflexions sur le fait social et la liberté individuelle

1. Les non-lieux peuvent être également des lieux de vie ou, en tout cas, des lieux de travail. Mais ils n'en restent pas moins non-lieux par leur organisation, destination et utilisation : il s'agit de gérer le transitoire ou, plus précisément dit, le temporaire; l'espace y est conçu pour absorber le temps, qu'il soit individuel ou collectif.

2. Voir le premier tome de La Règle du jeu, précisément intitulé Biffures (Leiris 2003). 
(Augé 2006). Donc «biffures» non seulement au sens littéral (ratures, rayures, gaps: le vers de Bérénice demeure incongru dans le contexte radiophonique et trivialement publicitaire de sa remémoration; la pause relationnelle dans les non-lieux ne va pas sans de sévères retouches identitaires à leurs entrée et sortie) mais au sens phonétiquement figuré, bifurs, dont Denis Hollier ${ }^{3}$ nous rappelle que, dans le langage de la signalisation ferroviaire, ce terme affiché sur un panonceau au-dessus des ballasts annonçait un aiguillage, une bifurcation, un changement d'orientation. Donc rectification et déviation : à une conception romantique, baudelairienne de l'expérience ethnographique qui a marqué la période de conquête de la discipline, se substitue une conception que j'appellerai brechtienne de celle-ci où, dans ce qui était déjà terme à terme contradictoire avec l'introduction et l'adoption parmi les principales consignes d'enquête anthropologique de la fameuse pratique de l'observation participante, l'accent se déplace de la participation à la distanciation, l'espace se courbe suivant le rythme des allers et retours entre la scène où se situerait l'autre et la salle où siègerait le soi. L'ethnologue se retrouve en réalité à égale distance des autres et de lui-même, tout comme le sont probablement ceux qu'il se propose d'étudier. Marc Augé (1988 : 116-117) rappelle, à ce sujet, que :

«l'anthropologie est toujours anthropologie d'une autre anthropologie. Le regard de l'ethnologue ne porte pas sur des réalités brutes qu'il serait le seul et le premier à découvrir comme problèmes : il rencontre des réalités sociales, pensées, élaborées, où ne se laisse pas lire simplement un rapport de force plus ou moins bien camouflé, mais un rapport de sens sans lequel la force ne signifierait rien et le camouflage encore moins ».

La scène sociale aura toujours quelque chose d' "épique» selon le mot de Bertolt Brecht, c'est-à-dire, comme le souligne Jean-Paul Sartre (1992 : 98), que tout individu qui y évolue et toute action individuelle qui s'y déroule " manifestent les grands courants sociaux et leurs significations »; "l'idéal du théâtre brechtien », poursuit-il (Ibid. : 110), «ce serait que le public fût comme un groupe d'ethnographes rencontrant tout à coup une peuplade sauvage. S'approchant et se disant soudain, dans la stupeur : ces sauvages, c'est nous ». Cet idéal, c'est la réalité de l'anthropologue, la stupéfaction en moins.

Il y a donc bien une valeur de présage dans l'alexandrin de Racine dont se souvient Marc Augé le matin du 20 juillet 1984, mais qui lui conférerait aussi, par antiphrase, une dimension jubilatoire. Au fond, l'ennui des voyages dont les non-lieux constituent l'une des cases départ aujourd'hui obligées n'est qu'affaire d'appareillage, nullement de pérégrination. L'embarquement sitôt effectué, la piste sitôt quittée, les amarres sitôt

3. Voir sa «Notice» sur Biffures in Leiris (2003: 1292). 
larguées, la barre sitôt franchie, c'est le regard qu'on porte sur soi, sur l'ici et sur l'ailleurs, qui change, et qui, comme l'écrit Leiris pour sa part (1973 : 126), fait que «nous nous ressentons intensément debout devant les choses ", lesquelles s'éloignant de nous s'éloignent de nos souvenirs au lieu de s'en trouver encombré et accablé. Cette «minute d'une plénitude déchirante» (Ibid.) qui est celle où l'on se sépare du quai ou du tarmac incite à prendre notre propre mesure et à espérer qu'au loin, plus tard, sur cette ligne inouïe de raccordement du temps et de l'espace qu'esquisse l'étrave du navire ou le nez de l'avion, une autre et nouvelle mesure de soi (et d'autrui) s'y substitue.

Ce n'est certainement pas sans raison qu'en 1922 le fondateur du surréalisme André Breton (1979: 108-110) avait sommé ceux qui souhaitaient le suivre dans sa rupture avec Dada pour aller «capter des voix d'outre-monde" (Bréchon 1971: 90) et se ressourcer, de lâcher tout (femme, enfant, maitresse, crainte et espérance, vie aisée, sans oublier la proie pour l'ombre) et de partir sur les routes ${ }^{4} .$. Si Breton, paradoxalement, ne le fit guère, l'ancien surréaliste Leiris, on le sait, s'y lança à corps perdu, et plus tard Marc Augé jusqu'à perdre haleine, comme l'auteur de Nadja le dit de toute vie valant d'être vécue, mais muni quant à lui d'un viatique d'ethnologue (ce que ne possédait pas encore Leiris à son départ), tous deux s'étant embarqués pour l'Afrique noire qui, dans notre culture en particulier française, fut d'abord et avant tout, et demeure peut-être si je me reporte à la belle expression d' "Afrique intérieure " forgée par Jean-Pierre Dozon ${ }^{5}$, une image mentale : une sorte de non-lieu

4. L’ouvre de Marc Augé présente quelques affinités avec le surréalisme non pas tant sur le plan de la démarche qui, pour l'essentiel, se veut et demeure scientifique, encore que l'approche esthétique et littéraire ne lui soit nullement étrangère (notamment Augé 1985, 1997, 2000, 2003a, 2007), qu'au niveau de ses champs de curiosité et d'investigation qui l'amèneront à fonder une anthropologie que je serais tenté de considérer aussi comme une poétique des mondes contemporains (Augé 1994b) : le langage, l'art, l'objet, le sacré, le rêve, le voyage, l'image, la mémoire, l'oubli, la modernité..., sans oublier ces fameux non-lieux auxquels les surréalistes eux-mêmes n'ont pas été insensibles bien que le mot qui sert à les désigner aujourd'hui leur manquât. Je me demande, en effet, si ceux qui se rallièrent très tôt au mouvement d'André Breton ne comptent pas parmi les premiers (tels Aragon, Perret, Desnos, Crevel, Soupault et Breton lui-même) à avoir exploré ce qui, à l'époque, put tenir lieu (si j'ose dire) de non-lieux, et pris conscience que dans ces entre-deux, l'identité, la subjectivité se trouvait elle-même placée en suspension: zones d'allées et venues, de circulations, de passages (comme ceux des faubourgs, comme les rues, ponts et marchés, comme les cafés, squares et bazars de Paris) où le flâneur surréaliste attendait que de l'insolite survînt, du sens surgît, ne serait-ce que sous le signe d'une rencontre (Nadja), dès lors en quête de "hasard objectif ". Le Paysan de Paris avait déjà quelque chose de l'ethnologue Augé "dans le métro " (1986).

5. Voir son introduction à la journée d'étude L'Afrique, miroir du contemporain, organisée par l'Institut interdisciplinaire d'anthropologie du contemporain (IIAC) le 19 juin 2007 à l'École des hautes études en sciences sociales. Je renvoie également à son ouvrage (Dozon 2003) qui propose une archéologie politique du « chassé-croisé singulier » entre une puissance impériale et ses colonies d'Afrique noire ainsi qu'une généalogie intellectuelle de leurs liens: peu de nations colonisatrices ont connu en effet, en leur sein, une telle présence africaine, du nom de la revue que le Sénégalais Alioune Diop fonda à Paris en 1947 (Ibid. : 227 sq.). 
symbolique ne serait-ce que par les multiples, parfois divergentes, représentations dont elle a été l'objet (par excellence terrain des biffures/bifurs), et que la "mode du négrisme", pour reprendre l'expression de René Depestre (1998: 79), allait propager pendant les années 1920 en l'associant expressément à un "dérèglement" du et des sens...

L'Afrique fut d'un coup haussée au niveau, d'une fable, d'une utopie, d'un mythe dont Roland Barthes (1957 : 239) rappelait qu'une des caractéristiques était de priver l'objet dont il parle de toute histoire: "Il n'y a plus qu'à jouir sans se demander d'où vient ce bel objet. Ou mieux, il ne peut venir que de l'éternité : [...] de tout temps, les "primitifs" ont préparé leurs danses en vue d'une réjouissance exotique ». Peut-être pourraiton voir là rétrospectivement un des points d'ancrage de ce qui a caractérisé pendant longtemps, au moins jusqu’à la fin des années 1940, l'approche et la construction des objets de l'anthropologie française africaniste, d'évidence plus portée que sa parente britannique (rappelonsnous le texte critique mais plein d'humour de la regrettée Mary Douglas $^{6}$ ) à décrire, exposer et analyser le foisonnement des mythologies africaines qu'à étudier concrètement les pratiques sociales, celles-ci étant parfois déduites de celles-là.

Surréelle, l'Afrique le fut probablement dans la mesure où elle permettait d'accéder à une autre réalité physique ou mentale, à un autre langage, à un autre espace/temps. Il faut reconnaître que la reprise théâtrale en mai 1912 des invraisemblables Impressions d'Afrique de Raymond Roussel avait ouvert la voie: Guillaume Apollinaire, Francis Picabia, Marcel Duchamp qui assistaient à cette représentation ne se trompèrent pas sur les nouvelles équations de faits et de mots $^{7}$ auxquelles pouvait donner lieu rien que l'évocation, serait-elle en l'occurrence pittoresque, de ce continent dont on supposa qu'y prévalait le "démon de l'analogie " et s'y ébrouaient des «bêtes animiques » comme le dira plus tard Blaise Cendrars (2005b : 242). Le noir auquel l'Afrique est d'ordinaire associée - couleur chère aux surréalistes qui y voyaient la métaphore des profondeurs cachées de l'individu, des courants souterrains de la création, des corridors de l'imagination, des effets de la magie tout autant que des attraits de l'humour (Bréchon 1971 : 88-90), et non plus seulement celle, nervalienne, du «soleil noir de la mélancolie »n'y est pas non plus pour rien... Mais l'Afrique devint également, et

6. Mary Douglas, "If the Dogon...", Cahiers d'études africaines, 1967, 28 : 659-672. Pour une synthèse sur cette question, voir l'article de Suzanne Lallemand : "Cosmologie, cosmogonie ", in Augé, ed. (1974 : 20-32), ainsi que les remarques de Luc de Heusch dans L'Homme (n 184, 2007 : 215-220).

7. Voir la "Présentation" d'Annie Lebrun (notamment pages 15 et 24) du livre posthume de Michel Leiris, Roussel \& Co, Paris, Fayard-Fata Morgana, 1998 : 15-61. 
paradoxalement, le modèle d'une modernité (ou plutôt d'une contremodernité) provocante, rebelle, transgressive, où les principes d'ordre et de coordination de l'existence sociale - de l'existence tout court - étaient supposés fondamentalement distincts de ceux qui, soi-disant guidés par la raison, l'action mécanicienne et le progrès technique, avaient conduit le monde occidental au bord du précipice. Aussi les lieux auxquels renvoient les adverbes de la sentence de Leiris devraient-ils être permutés. Car c'est bien ici qu'on s'est enlisé, non point dans les déserts de l'Orient ou d'ailleurs (je reviendrai sur ce point plus bas).

\section{Génie nègre}

Le tête-à-tête encore ensommeillé avec soi-même (Augé 1985: 23) mais déjà puissamment infiltré par le langage (et par la culture) dont témoignent la phrase de Michel Leiris et l'alexandrin remémoré par Marc Augé donnerait quelque réalité sensible, en l'occurrence subjective, à la fameuse proposition de Claude Lévi-Strauss qui, dans son introduction à Sociologie et Anthropologie de Marcel Mauss (1950: XLVII), soutenait qu'à l'échelle de l'histoire de l'humanité " le langage n'a pu naître que tout d'un coup ». "Les choses ", ajoutait-il, «n'ont pas pu se mettre à signifier progressivement. [...] Un passage s'est effectué, où rien n'avait un sens, à un autre où tout en possédait.» Il y aurait, commente Marc Augé (1994a: 16-17), comme une antériorité du symbolique sur la connaissance. Il a fallu que, dès l'apparition du langage, l'univers signifiât, "c'est-à-dire », précise-t-il (Augé 1988 : 63), «bien avant que les capacités de la connaissance pussent répondre à l'exigence de sens. L'univers fait signe donc et nous admettrons [...] que le sens de ces signes a procédé initialement d'une urgence soudaine concomitante au surgissement du langage et sans commune mesure avec le rythme progressif et continu de la connaissance ». Transposée au niveau de l'histoire des idées (idées sur..., plutôt qu'idées de...) et, en particulier, au niveau de celle des représentations, cette proposition garde sa valeur heuristique : on peut y dénicher maints exemples de ce décalage à la fois temporel et logique entre, mettons, donner sens et prendre connaissance; peut-être est-ce ce qui la caractérise en partie, de sorte qu'on serait tenté d'appliquer la notion d'idéologie aux configurations symboliques (mythification impliquerait mystification) ou, plus généralement dit, aux processus de symbolisation (Augé $1977: 83$ sq.) que cette histoire-là révèle et met en œuvre.

C'est au décryptage d'un de ces processus et moments que je voudrais m'attacher dans la suite, faisant l'hypothèse, si je reprends le titre de cet article qui m'est venu également du fond de la conscience encore ensommeillée, que l'Afrique semble bien avoir été en tête comme cela peut aussi se 
dire, au-delà de la métaphore sportive et particulièrement cycliste, d'un banal papier à lettres : tout à la fois image, logo, symbole ou rien qu'un nom dès lors réduit à la fonction d'un signifiant flottant dont l'oscillation se vit accrue par la vogue (on aimerait écrire la vague) d'un art qui lui fut attribué avant que d'être vraiment documenté, et que, de façon certes impropre et immodérée mais en partie entretenue sinon amplifiée par l'engouement sans précédent qu'il allait susciter aux lendemains de la Première Guerre mondiale, notamment en Europe occidentale, on qualifia de nègre. - Terme élastique et excessivement coloré ${ }^{8}$ dont il faut peut-être rechercher l'origine et la légitimité esthétique de son emploi d'abord séditieux, comme de sa fortune paradoxalement avant-gardiste, chez le Rimbaud d'Une saison en enfer (nombre d'artistes et de poètes du début du XXe siècle lui reconnaîtront à cet égard leur dette) où celui-ci s'en sert pour désigner et justifier son rejet de la raison occidentale et de la morale chrétienne?

Ainsi mise en tête, en exergue, en enluminure (ne serait-ce que par sa couleur) - ce qui signalait métaphoriquement son incrustation dans l'esprit du temps (dans la tête) et ce qui venait en dessiner l'arborescence (à la tête) -, l'Afrique signifia avant qu'elle ne fût réellement connue. Et même si elle l'était quelque peu, la frontalité de son art que, de manière ethnographiquement floue mais esthétiquement importante, Carl Einstein (1961) avait mise en évidence, amènerait à penser - osons le jeu de mot - qu'elle fut d'abord connue de tête, ou du moins que c'est par la tête qu'on la reconnaissait comme le laisse entendre cette remarque de Tristan Tzara écrite en 1917 (1975 : 394-395) : l'artiste nègre, observait-il, «concentre sa vision sur la tête, la taille dans du bois dur comme le fer, patiemment, sans se soucier du rapport conventionnel entre la tête et le reste du corps. Sa pensée est: l'homme marche verticalement, toute chose de la nature est symétrique. En travaillant, les relations nouvelles se rangent par degrés de nécessité ; ainsi naquit l'expression de la pureté».

L'Afrique devint même - je l'ai suggéré - l'emblème sinon le simulacre d'une modernité artistique, subversive autant que festive. Au cabaret Voltaire de Zurich d'où allait émerger le mouvement dada, Hugo Ball et Tristan Tzara avaient organisé en 1916 des soirées placées sous le signe de

8. Sous l'étiquette d' "art nègre ", et outre les statues et masques d'Afrique noire proprement dits, étaient incluses les sculptures d'Océanie et de la Côte Nord-Ouest américaine aussi bien que les compositions de ragtime, les mélodies, harmonies et cadences du jazz, les danses afro-brésiliennes, les poésies, légendes et cosmogonies du sud du Sahara ou de l'intérieur de l'Australie, la littérature de la Harlem Renaissance, etc. (Clifford 1993 : 844).

9. Rappelons-nous la phrase de Rimbaud: "Je suis une bête, un nègre [...] J'entre au vrai royaume de Cham [...] Plus de mots. J'ensevelis les morts dans mon ventre. Cris, tambour, danse, danse, danse, danse!» (Une saison en enfer, Mauvais sang in Euvres, Paris, Garnier, 1960 ; éd. de Suzanne Bernard). 
rythmes, danses et chants nègres (des «nègreries») qui ne sont pas sans rappeler les saynètes popularisées à la fin du XIXe siècle par les minstrels nordaméricains où des Blancs se grimaient en Noirs, où des Noirs se présentaient plus noirs que noirs en se charbonnant la face (Jamin \& Séité 2006; Béthune 2007), sauf que l'accent fut mis ici moins sur la pantomime que sur la déclamation de " poème nègres " traduits, copiés ou inventés - sortes de primitive-made - dont était placardée la structure phonétique, soulignée l'écriture asyntaxique presque plastique, et intensifiée la vocalisation supposée incantatoire (Fauchereau 1976: 215 sq. ; Blachère 1981 : 140 sq. ; Clifford 1993 : 846). Il fallait réapprendre à "danser avec la langue ", lâcha Blaise Cendrars dans un poème écrit en 1916 (2006a : 134). Comme si, en privilégiant le son et le rythme, la frénésie et la fantaisie, l'émotion et la participation, ainsi qu'était censé le faire le monde noir - théâtre du primitivisme du sens, règne de la primauté des sens (Adotevi 1998: 44 ; Somé 1998: 219 sq.) - devaient s'ensuivre non seulement un renouveau de l'activité poétique mais un retour de l'art dans la vie, du rêve dans la pensée, du geste dans le langage, de l'imaginaire dans le réel, de la magie dans l'objet... Il s'agissait de restaurer un rapport sensible et sensuel entre l'homme et la nature, rapport que le "penser-dirigé " (Tzara 1981 : 269) de la civilisation occidentale, le credo de sa religion et l'académisme de son art avaient figé, cloisonné, puis distendu, brisé et vicié.

Du fait de l'«authenticité », de l'"altérité originelle», des licences de tous ordres et de la "réelle présence " des idoles de toutes espèces dont on le croyait nanti, le monde noir fut érigé en modèle à rêver sinon à suivre (Blachère 1981: 164 sq.). Le poète, l'artiste, l'homme n'allait plus se mesurer seulement à lui-même ni se soumettre stupidement à un seul dieu, à un seul canon ou à une seule nation: toutes limites franchies et toutes frontières rompues, il devait se confondre «avec le bric-à-brac illimité de l'univers» (Leiris 1992b : 111). Dada trouva bien quelque génie au paganisme dont les gens d'Afrique, en transmutant un art de voir et d'entendre en un art de vivre (Augé 1982 : 11), étaient vus comme les premiers (et derniers) fidèles. Mais si, remarque par ailleurs Marc Augé (1979 : 158), "la découverte de l'art nègre " et plus tard "celle des systèmes dits philosophiques de l'Afrique a eu une signification progressiste - manifestant que ceux que l'Occident colonisait et dont il se servait notamment pour faire la guerre n'étaient pas des "sauvages" ", - leur diffusion dans le tissu artistique et social de l'époque ne se fit pas sans malentendu ni aveuglement en partie dus à cette mythification/mystification que j'évoquais plus haut et qui, pour qu'elle fonctionnât à plein régime, supposait (nécessitait ?) que la situation économique, la condition sociale et les variations historiques des peuples du continent noir fussent je ne dis 
pas tues mais mises entre parenthèses. Était-ce là le prix à payer pour que l'art s'exotise et, s'exotisant, vienne régénérer, comme par un effet de boomerang, les manières de se représenter le monde et d'agir sur lui ? Le fait est que la plupart des artistes et poètes qu'on peut qualifier de négrophiles ne se préoccupèrent aucunement, et ce jusqu'à la fin des années 1920, de la réalité sociale et politique des gens de couleur qu'ils avaient pris pour exemples, parfois comme parangons, pas plus qu'ils ne s'intéressèrent aux significations de leurs systèmes symboliques dont, au gré de leur inspiration ou de leur fantaisie, ils prélevaient ici ou là, telle une matière première, un simple élément : sculpture, mythe, conte, légende, rythme ${ }^{10}$.

Bien qu'elle fût intimement énoncée et idiosyncrasiquement glosée, la sentence de Leiris que j'ai citée au début de cet article pourrait avoir une portée plus générale (historique, sociologique, anthropologique) qualifiant les rapports ambigus que, précisément, les avant-gardes artistiques occidentales, en particulier françaises, ont noués dès le début du XXe siècle avec l'Afrique noire, du moins avec les images qu'elles en retinrent, les objets qu'elles en récupérèrent, les mots et tournures qu'elles lui empruntèrent, en somme avec les masques, fétiches et voix de l'Afrique. Devenues ici, en Europe, fruits à la tête dans la mesure où ces figures d'un autre monde et d'autres peuples furent parfois plantées sur le chevalet des créations plastiques les plus sophistiquées et les plus dérangeantes (certaines œuvres cubistes de Braque, Picasso ou Gris ne se privèrent pas de le faire), conviées au chevet de poètes des "recoins ignorés de l'âme" comme eût dit Apollinaire (tel Tzara, tel Cendrars), ou convoquées au pupitre de musiciens en rupture avec la mesure à trois temps et le système tonal dit tempéré (Debussy, Satie, Stravinsky, Ravel, pour ne citer qu'eux), il n'en reste pas moins que là où elles étaient produites et d'où elles étaient arrachées, les nations européennes s'élançaient, virevoltaient, pataugeaient dans le découpage de frontières, le partage d'influences, le déplacement de populations et, surtout, avaient tenu et tenaient encore les corps et les esprits - les «têtes» - en asservissement.

10. Ni Debussy ni Poulenc qui composèrent pourtant les premières pièces où le terme nègre apparaît dans le titre - Le Petit Nègre pour l'un (1909), qui redouble le célèbre Golliwog's Cake Walk des Children's Corner de 1908, la Rhapsodie nègre pour l'autre (1917) aux accents d'ailleurs plus orientaux qu'africains ou jazzy; ni Braque ni Picasso dont le goût pour l'art nègre s'affirma très tôt et s'exprima plastiquement (Les Demoiselles d'Avignon datent de 1907); ni Apollinaire ni Cendrars non plus que Tzara qui s'extasiaient devant les idoles et fétiches africains - ces "Christs des obscures espérances » - ; aucun donc ne manifesta la moindre curiosité ethnographique pour des peuples dont ils attendaient pourtant que leurs productions artistiques vinssent régénérer le langage poétique ou musical, la vision du monde, la perception des choses et la représentation du corps. Â la notable exception peut-être d'un musicien comme Darius Milhaud qui prêtera à ces peuples une attention soutenue - je le montrerai plus loin - mais dans un contexte géographique et sociologique totalement différent. 
Cependant, au là exotique et menacé d'enlisement allait se substituer un ici où l'on s'enlisa bel et bien jusqu'au cou: dans les tranchées et casemates de la Grande Guerre. Au lieu de fruits à la tête, c'étaient des balles et des éclats d'obus qu'on risquait de récolter en pleine face, et, au lieu d'odeurs fruitées, à des gaz paralysants qu'on s'exposait. Non seulement des vies furent détruites par millions, des "gueules cassées " par milliers, des membres disloqués, des poumons ravagés, mais l'esprit traversa une de ses crises les plus profondes comme le déplora Paul Valéry dans ses deux célèbres lettres de 1919 expressément consacrées à la crise de l'esprit: "L'oscillation du navire a été si forte", concluait-il (2005: 17), "que les lampes les mieux suspendues se sont à la fin renversées ». La barbarie, la sauvagerie, la "déraison " n'étaient pas là où l'on crut qu'elles se trouvaient à l'état natif. Aussi la sentence de Leiris pouvait-elle vraiment s'inverser, le paradoxe étant que les «fruits exotiques" ne connurent ici, comme par une sorte d'expiation, leurs pleines croissance et expansion qu'à la fin de ce premier grand conflit mondial, au cours de ce qu'on a nommé les Années folles et que la langue anglaise, souvent plus limpide, a baptisées les Roaring Twenties, lesquelles s'échelonnent grosso modo de 1919 à 1929.

Au reste, c'est à Leiris (1996: 1125-1159) qu'on doit une des études les plus pénétrantes et les mieux documentées sur la vogue de l'art nègre et de la mythologie africaine qui apparut et se répandit avec fièvre sinon ferveur pendant toute cette période. Placée en ouverture de Afrique noire: la création plastique, elle est intitulée "la "crise nègre" dans le monde occidental ", faisant ainsi directement référence à un mot de Jean Cocteau qui eut beau jeu toutefois d'appeler « crise » l'engouement que lui-même avait éprouvé et contribué à faire joyeusement et frivolement partager sur les scènes et dans les boîtes parisiennes à la mode...

Je ne reprendrai ni le cadre ni les termes de l'analyse de Leiris, dont l'ampleur historiographique dépasse les limites d'un article, mais je chercherai plutôt à rendre compte d'un événement artistique et culturel : la représentation en 1923 d'un "ballet nègre " mimant la genèse du monde selon des mythes et légendes africaines, et qui est quelque peu oublié aujourd'hui bien que ce spectacle ait représenté une « date importante dans l'histoire de la diffusion de l'art nègre en France » comme le signale Leiris sans cependant, sur ce point précis, expliciter son jugement ni fouiller la comparaison qu'il amorce (ce qui venant de lui est pour le moins curieux). En effet, il se contente d'écrire (Ibid. : 1156) : « une grande soirée parisienne était placée esthétiquement sous le signe de la mythologie africaine tout comme le 29 mai 1913, avec l'œuvre fameuse d'Igor Stravinsky Le Sacre du printemps, il en avait été des rites de l'Europe [sic] païenne dans le cadre des Ballets russes 
de Serge Diaghilev ». - Pendant, en quelque sorte, du primitivisme exotique dont l'Afrique était considérée non seulement comme la matrice originelle mais aussi comme la scène contemporaine dans la mesure où étaient censés toujours s'y nouer des rapports analogiques entre l'imaginaire et le réel, s'y dérouler tout un jeu de correspondances symboliques entre les séquences mythiques ou rituelles et les divers domaines de la vie sociale et matérielle.

M'invite également à envisager cet événement artistique la contiguité sémantique du nom de ce ballet ( $L a$ Création du monde) avec le titre que donna Marc Augé en 1974 à un recueil de textes africanistes sur la religion, les représentations, l'idéologie ( $L a$ Construction $d u$ monde) où il était question entre autre de comprendre comment un monde social peut se construire, ou se reconstruire "lorsque l'agression extérieure renverse ses évidences anciennes» (je souligne), comme cela est annoncé dans le prière d'insérer dudit ouvrage (premier d'une collection, « Dossiers africains », que Marc Augé venait de fonder avec Jean Copans aux Éditions François Maspéro). Ce qui, toute proportion gardée, fut le propos en l'occurrence esthétique des auteurs de La Création du monde et, notamment, de Blaise Cendrars (1887-1974) qui en a été le principal artisan, lui qui eut le bras droit amputé au niveau du coude - son bras d'écrivain - après avoir reçu un éclat d'obus lors de l'offensive de Champagne en septembre 1915, soit un an presque jour pour jour après son engagement dans la Légion étrangère. Fernand Léger (1881-1955), son ami depuis 1912, à qui Cendrars fera appel pour dessiner et peindre les décors, le rideau de scène et les costumes du ballet qu'il avait en tête, avait été lui-même grièvement gazé alors qu’il était brancardier sur le front de Verdun en $1916^{11}$. Il y avait de quoi vouloir repenser le monde, et d'abord son propre rapport au monde. Certes, les idoles et fétiches nègres ne rigolent pas souvent, notera Cendrars (2005b : 270), «mais les machines jamais qui grincent des dents. C'est de l'épouvante. Elles sont beaucoup plus féroces et revendicatrices que Jéhova, le Dieu vengeur des Juifs. Voyez notre outillage de guerre, il extermine tout avec indifférence et jusqu'à la dernière et ultime génération ».

\section{Un ballet nègre et cubiste}

C'est étrangement à travers l'évocation d'un souvenir d'enfance traumatisant que Cendrars a parlé de sa «découverte» de l'Afrique noire et

11. Tous deux évoqueront leur expérience du front dans des œuvres majeures: L'Homme foudroyé et La Main coupée pour Cendrars, La Partie de cartes pour Léger où l'on voit des hommes tubulaires, robotisés, des poilus, - démantèlement des masses corporelles en des assemblages géométriques (cônes, cylindres, cubes) qui apparente de fait ses toiles de l'époque aux visées cubistes, c'est-à-dire à la représentation non point de l'espace mais du volume, non point de ce qui est perçu mais conçu ou imaginé (voir Kahnweiler 1946: 109 sq.; 1963: 51-56). 
retracé la naissance de sa passion pour l'art et la littérature des Nègres, qui deviendront le "réceptacle de ses rêves et de ses terreurs nocturnes" comme eût dit Denise Paulme (in Paulme \& Brosse 1956: 5).

Dans Le Lotissement du ciel (2005: 257 sq.), Cendrars raconte comment, tout jeune garçon ne sachant pas encore lire, il s'introduisait en catimini dans la bibliothèque de son père et faisait basculer d'un des rayonnages du bas un fort volume in $-4^{\circ}$ de la Nouvelle géographie universelle d'Élisée Reclus : le tome IX consacré à l'Afrique équatoriale qui, en tombant sur lui qui n'était guère plus haut qu'une botte, s'ouvrait toujours à la même page, "sur une gravure représentant une grande idole de bois accroupie au pied d'un arbre géant dans la forêt vierge, une idole cubique aux yeux démesurés, aux dents grimaçantes, ce qui me terrorisait, et je me sauvais au bout du corridor, chancelant, claquant des dents, moite de frayeur, puis je revenais sans bruit sur mes pas...» (ibid.: 258)... "L'idole de bois avait deux seins. En y regardant de près elle était aussi pourvue d'un sexe masculin, un pilon en battant de cloche [...]», et « la nuit, mon angoisse, mes cauchemars devenaient obscènes" (ibid.: 262). Ce fut là l'origine de sa passion pour l'art nègre qui ne s'est jamais apaisée depuis, "pas plus que ne s'apaisa jamais cette titillation intime" qu'il éprouvait à la vue d'une statuette ou d'un masque nègre, "rappel lancinant", observet-il (Ibid.: 263), "de l'idole qui me terrorisait dans mon enfance et me possédait la nuit ».

Mais, comme souvent chez Cendrars, le récit, aussi détaillé soit-il, aussi autobiographique se prétend-il, n'est pas l'exact reflet de la réalité ni la copie fidèle du passé : il n'existe pas de tome IX de la Nouvelle géographie universelle d'Élisée Reclus consacré à l'Afrique équatoriale, pas plus qu'il n'existe dans les dix-neuf volumes de l'œuvre (non point vingt-quatre comme il l'écrit), une gravure $\mathrm{d}^{\prime}$ " idole nègre " répondant à sa description voulue pourtant précise, et dont, à l'adolescence, il constatera d'ailleurs que quelqu'un (pas lui) l'avait arrachée du volume en question (ibid. : 262). La besogne de la mémoire et la lecture du texte qui s'évertue à la transcrire ne sont jamais achevées, et "en cet inachèvement réside l'essentiel ", remarque Patrick Williams (1989 : 80-81), où non seulement se fait « entendre le rire du créateur " mais s'échafaude sa mythologie personnelle propice à toutes sortes de versions, transformations et déformations, si ce n'est propice à l'évasion : "il faut inventer sa propre grille " ${ }^{12}$. Les récits de souvenirs d'enfance - fussent-ils faux souvenirs, comme celui de Cendrars, ou comme celui de Leiris, qui inaugure la somme de La Règle du jeu (un petit soldat de plomb ou de carton-pâte, cette autre idole et relique de l'enfance, tombant 
soudain d'une table sur le plancher sans se casser ${ }^{13}$ ) -, seraient une des voies par laquelle paradoxalement la subjectivité s'émanciperait d'elle-même, par la langue, par l'écriture qui introduit de fait une distance de soi à soi tout en instituant un rapprochement, une complicité, un partage avec l'autre, le lecteur (Augé 2006 : 62-62). Sur ce sujet, Cendrars, qui en 1912, changea son nom d'état civil, Frédéric Louis Sauser, en celui de Blaise Cendrart puis Cendrars, en connaissait manifestement un bout, ne serait-ce qu'en s'inventant ce nom de plume autant inscriptible qu'au sens propre volatil. Dans l'antichambre des éditions de La Sirène qu'il fonda en 1919 et où sera publiée sa célèbre Anthologie nègre (2005a), de petits cadres en bois cloués au mur signalaient le nom, les jours et heures de réception du gérant, de l'administrateur et du directeur littéraire ; sur celui qui lui était destiné en tant que fondateur aurait été inscrit : "Monsieur Cendrars n'est jamais là »! (Guiette 1994 : 49).

Chez Cendrars, il semble évident que l'Afrique, avec ses rites et ses cosmogonies païennes s'accordant dès lors à ceux et celles qu'il s'invente, ou les réfléchissant par la recomposition permanente que leur mode de transmission essentiellement oral permettrait, est d'abord cosa mentale ${ }^{14}$, affaire d'image, de langage et d'écriture. Au reste, lui, le bourlingueur, le baroudeur, n'y mettra jamais les pieds si ce n'est lors d'une brève escale à Dakar en 1924 sur le chemin du Brésil. La gravure de Reclus que Cendrars nommera dans la suite l'IDOLE ainsi composée en petites capitales - faux souvenir donc, ou comme dirait Freud souvenir-écran -, prend la même valeur que la sentence de Leiris ou le vers remémoré d'Augé. C'est une image-présage qui s'offre à toutes les biffures/bifurs possibles mais qui n'en reste pas moins constellée de vues, de chimères, de rêves d'un autre ordre et d'un autre monde: un bourgeonnement foisonnant (fruit à la tête), quels qu'en soient les racines, le terreau, le lotissement - bien céleste celui-là - où se confondent l'ici et là. Elle amènera en tout cas Cendrars à imaginer une nouvelle création et représentation du monde à partir de contes, légendes et mythes africains qu'il a patiemment recopiés à la Bibliothèque nationale (avec l'aide de Raymond Radiguet ${ }^{15}$ ), puisant aux meilleures sources (Henri-Alexandre Junod, Leo Frobenius, Édouard Jacottet, Maurice Delafosse, Henri Trilles notamment), et qu'il a "retravaillés",

13. Voir le chapitre «...Reusement!» du premier tome, Biffures (Leiris 2003: 3-6) et le commentaire qu'en fait Denis Hollier (Ibid.: XXIII-XXV) montrant comment l'écriture est au fond parade: «manière, sans doute de parader, mais d'abord manière de se parer contre les risques associés à la communication en direct ».

14. Voir la préface de Christine Le Quellec Cottier à Anthologie nègre de Cendrars (2005a : X).

15. Ibid.: XV. 
parfois reformulés, organisés thématiquement - suivant en cela la classification de l'administrateur des colonies François-Victor Équilbecq publiée en $1913^{16}$ - pour en extraire la seule veine poétique sans donc se soucier de découpage ethnolinguistique ni se préoccuper des aires socioculturelles ou des références ethnographiques ${ }^{17}$. Ce sera Anthologie nègre qui connâ̂tra un succès d'estime dans le milieu artistique et littéraire de l'époque. Quelques-uns de ces textes, notamment extraits de la première partie "Légendes cosmogoniques ", vont lui servir de canevas pour écrire l'argument de La Création du monde ${ }^{18}$, et, en particulier, un conte d'origine fang tiré du recueil du missionnaire Henri Trilles Contes et légendes fang du Gabon paru en 1905 à Neuchâtel ${ }^{19}$ (voir Annexe I).

La première représentation du ballet eut lieu à Paris le 25 octobre 1923, au théâtre des Champs-Élysées, en seconde partie d'un spectacle où fut également donné un sketch musical Within the Quota, - évocation parodique de l'american way of life (avec projection cinématographique, fox-trot et ragtime) et premier "ballet jazz ", a-t-on dit abusivement, qui avait été orchestré par Charles Kœchlin (Caillet 2001 : 117) à partir d'une composition pour deux pianos de Cole Porter ${ }^{20}$. La chorégraphie de La Création du monde fut réglée par Jean Börlin (1893-1930), le danseur étoile des Ballets suédois qu'avait fondés en 1920 le riche aristocrate et industriel suédois Rolf de Maré (1888-1964), grand amateur et collectionneur de peinture moderne et d'art primitif, installé à Paris dans un appartement que décora Fernand Léger (à qui de Maré avait d'ailleurs

16. Voir la réédition par Robert Cornevin des Contes populaires de l'Afrique occidentale, précédés de Essai sur la littérature merveilleuse des Noirs, de François-Victor Équilbecq (Paris, Maisonneuve \& Larose, 1972).

17. Ce qui pourrait expliquer la retenue de l'«ethnologue» Leiris que j'ai mentionnée plus haut, outre les réserves idéologiques et même littéraires que celui-ci a souvent manifestées à l'égard de Cendrars, probablement trop anarchiste et prolixe à ses yeux. Dans son Journal, Leiris (1992a : 150) note pourtant ceci, il est vrai quelque peu désabusé à ce moment-là (mai 1929) et ne faisant allusion qu’aux Dix-neuf poèmes élastiques de Cendrars publiés en 1919: "J’en arrive à préférer la lecture de Cendrars à celle des livres surréalistes, car chez Cendrars, si mauvais que cela soit, on trouve du moins une certaine vie. Dans les Dix-neufpoèmes élastiques, il y a même des choses très touchantes, telles que : "C'est la première fois que je voudrais être femme - être femme et s'ouvrir à l'avenir enfantin" ". C'est là une de ses très rares allusions à Cendrars. Par ailleurs, Leiris n'a probablement assisté à aucune des représentations de La Création du monde, alors que, dans La Règle du jeu (2003), il évoque longuement celle de Parade (ballet de Jean Cocteau sur une musique composée par Erik Satie, avec des décors et costumes dessinés par Picasso et une chorégraphie réglée par Léonide Massine, qui fut créé à Paris, au théâtre du Châtelet le 18 mai 1917 ; voir infra), et dont Leiris a vu une reprise en 1923 au théâtre des Champs-Élysées.

18. Quatre ans auparavant, en juin 1919, Cendrars avait déjà utilisé l'un de ces textes pour un court spectacle intitulé «La légende de la création » dont il régla la mise en scène et l'éclairage, et qui faisait partie de la Fête nègre, une manifestation organisée par le collectionneur, marchand et critique d'art Paul Guillaume, au théâtre des Champs-Elysées.

19. Réédité chez Karthala, Paris, en 2003. 
acheté quelques tableaux). Sous l'administration de Jean Hébertot, il finança la troupe jusqu'en 1925, la propulsant, non sans de vives controverses, à l'avant-garde des manifestations artistiques de la capitale ${ }^{21}$.

Tout en "jetant aux oubliettes la tradition chrétienne " ${ }^{22}$ au profit d'une cosmogonie et d'un rituel païens, les auteurs de La Création $d u$ monde allaient en outre révolutionner l'art du ballet dont Jean Cocteau disait qu'il devait être le lieu par excellence où peuvent s'assimiler et s'unifier tous les arts, tous les registres et tous les répertoires, l'orchestre et la parole, la chanson populaire et l'air d'opéra, la poésie, la danse, l'acrobatie, le cirque, le drame, la pantomime, la satyre, etc. (Pastori 2005 : 31). - Lieu qui ainsi pensé et réellement pratiqué n'était pas loin d'apparaitre, au regard du goût et des conventions de l'époque, sinon comme un non-lieu du moins comme un non-sens artistique où le mélange et l'excès de genres pouvaient heurter les mœurs, et pas seulement celles des académies. Satie, Picasso, Cocteau en avaient déjà fait les frais avec le ballet Parade, représentation qui déclencha un tel tollé après la Première du 18 mai 1917, en "pleine boucherie de Verdun" et mutineries (Richardson 2007 : 41 sq.), que Satie, qui avait introduit dans sa musique des bruits de sirène, des cliquetis de machine à écrire, des coups de feu et des clapotis de bassine d'eau (pour le fameux Ragtime du paquebot), faillit être jeté en prison à la suite d'une plainte qu'avait déposée un journaliste ombrageux et réactionnaire, Jean Poueigh, pour avoir été traité publiquement par lui de "trou du cul ", et même de "trou du cul sans musique ${ }^{23}$...

La Création du monde ne fut pas mieux accueillie par la critique, spécialisée ou pas. Un chroniqueur du journal L'Euvre écrivit le 27 octobre 1923, soit deux jours après la Première, que le ballet par lui qualifié de cubiste "a des intentions symboliques puisqu'il s'agit de nous montrer le monde au sortir du chaos ", mais, ajouta-t-il non sans humour, on peut craindre "à voir çà qu'il n'y soit déjà rentré " (in Abramovic \& Hergott

20. Il allait devenir au cours des années 1930 l'un des plus célèbres auteurs-compositeurs américains de comédies musicales et de chansons que nombre de musiciens de jazz inscriront à leur répertoire des standards (Williams 2006 : 32 sq.), tels que Night And Day, What Is this Thing Called Love, Love For Sale, All OfYou, etc. Comme Cendrars, Cole Porter (1891-1964) était un « ancien de la Légion». S'étant engagé à la fin de 1917, il fut affecté au Régiment de Marche de la Légion étrangère (RMLE) puis à l'école d'artillerie de Fontainebleau. Après la guerre, il séjourna quelques temps à Paris, où, à côté d'une vie mondaine et tapageuse, il suivit des cours de musique à la Schola Cantorum.

21. Les Mariés de la Tour Eiffel de Jean Cocteau et des musiciens du "Groupe des Six " (Arthur Honegger, Germaine Taillefer, Georges Auric, Darius Milhaud, Francis Poulenc, Louis Durey) en 1921, puis, en 1922, L'Homme et son désir de Paul Claudel et Darius Milhaud, et Skating Rink de Riciotto Canudo et Arthur Honegger, Relâche de Francis Picabia et Erik Satie en 1924, comptent parmi les représentations les plus marquantes et controversées de cette troupe.

22. Christine Le Quellec Cottier, préface à Anthologie nègre de Cendrars (2005a : XXII).

23. Voir également Pierre Bergé, Album Cocteau, Paris, Gallimard, 2006 : 96-99. 
2000 : 114). Au moins ce critique anonyme avait-il mis involontairement le doigt sur une des composantes essentielles du ballet où furent privilégiées, sous l'influence certaine de Fernand Léger, la dimension plastique des figures dansées et les évolutions heurtées ou glissées, presque mécanisées, des artistes dansants. En somme, se rappelle Léger (2004: 117), il s'était agi de "rompre entre le visuel de la salle et celui de la scène, faire disparaitre l'individu pour utiliser le matériel humain. Créer une scène d'invention. Le matériel humain apparaît, mais égal comme valeurspectacle à l'objet et au décor ». Dissimulés sous des maquettes inspirées de statues et masques africains principalement d'origine baoulé, sénoufo (Côte-d'Ivoire), bambara (Mali) et kota (Gabon) représentant des bêtes, des plantes, des humains ou des divinités, les pieds bien à plat sur le plateau de scène (pas question de faire des "pointes") ou fixés sur des échasses, le corps revêtu de lourds accoutrements, ou plaqué à même le sol, rampant derrière des silhouettes animales ou végétales peintes, les danseurs évoluaient de façon délibérément décalée par rapport à la partition de Darius Milhaud ${ }^{24}$, qui, dès lors, s'entendait plus comme un contrepoint musical de l'argument du ballet qu'elle ne remplissait la fonction classique de stimulateur ou d'accompagnement de la danse.

En outre, rehaussé par tout un système d'éclairage que Cendrars avait conçu et réglé (Abramovic \& Hergott 2000 : 94), le spectacle, grâce à la patte de Léger, put être assimilé à un tableau cubiste animé où prévalait non point l'expressivité de l'art nègre bien sûr pris pour modèle, ni sa prétendue magie, mais où, par les décors, les maquettes et les costumes, par leur disposition scénique, étaient mis en avant l'agencement géométrique de ses formes (cubes, cônes, cylindres, trièdres), le registre de ses couleurs (noir, blanc, bleu nuit, ocre clair et foncé), et la cinétique de son exposition, c'est-à-dire la dimension spatiale et par conséquent mobile de ce qu'on pensait être l'un des traits majeurs de sa création, de sa plastique et de son exhibition sociale et rituelle.

Bien que Léger ne fût guère féru d'art nègre à la différence de Braque et

24. De quelques années plus jeune que Cendrars et Léger, Darius Milhaud (1892-1974), par ailleurs de santé fragile, ne sera pas mobilisé pendant la Première Guerre mondiale. Son expérience est essentiellement "exotique » : le Brésil où, en tant que secrétaire de Paul Claudel nommé comme ambassadeur, il séjourne de 1916 à 1918, et découvre les rythmes des maxixes et sambas (les pièces musicales Le Bouf sur le Toit, opus 58, et Saudades Do Brasil, opus 67, en portent la marque) ; les États-Unis, à l'automne-hiver 1922, où, à Harlem, il se familiarise avec les cadences, accents et notes plaintives du blues et du jazz. En 1923, à 31 ans, Milhaud qu'un critique musical, Henri Collet, associa au groupe dit des six (voir supra, note 21), s'était déjà rendu célèbre par ses musiques de scène, surtout par les scandales auxquels elles donnèrent lieu: Le Boeuf sur le Toit, sur un argument de Jean Cocteau, avec des décors de Raoul Dufy en 1919 (opus 58); L'Homme et son désir, ballet sur un argument de Paul Claudel avec des décors d'Audrey Parr, qui fut composé en 1918 (opus 48) et représenté en 1921 par les Ballets suédois. 
de Picasso, et qu'il s'en tînt à une connaissance qu'on peut estimer superficielle de ses objets (l'expérience de La Création du monde restera unique dans son œuvre), s'étant contenté de relever quelques croquis d'après des illustrations de la Negerplastik de Carl Einstein, des œuvres de la collection d'art primitif de Paul Guillaume ou des vitrines du Musée d'ethnographie du Trocadéro, c'est avec une remarquable intuition et un non moins remarquable savoir-faire que le peintre a perçu et restitué l'essence de cet art qui est tout de volume, d'espace, de mouvement: un "art objet», observait Daniel-Henry Kahnweiler (1963: 225); l'artiste africain crée en effet, insistait-il (ibid. : 226), de « vrais objets qui, pouvant se placer n'importe où, ne demandant ni base ni socle, ne se rattachant à aucune architecture préexistante, sont sculpture vraie, souverainement ".

En ce sens, Léger avait parfaitement compris que les dieux d'Afrique noire qu'il avait cherché à représenter - sorte de mâts-totems, au contresens ethnographique près - par trois figures monumentales au visage en forme de masque (les dieux avancent toujours masqués mais ne sont pas des "dieux cachés ») étaient des "dieux-objets " (Augé 1988) où le signifiant collait au plus près de signifié, et que l'on pouvait manipuler, actionner, déplacer à loisir sur la scène. On pouvait même les associer, sans rupture de ton ni souci d'échelle ou de vraisemblance (un éléphant restait suspendu en l'air au côté d'une déité) à d'autres figures disposées " en batterie » dans un rapport de contiguïté, comme il en est du Komo des Bambara " où la puissance est d'entrée de jeu investie dans une diversité d'objets et non dans une "chose-dieu" unique" (Colleyn 2004 : 70) ${ }^{25}$. C'est cela aussi le génie du paganisme : un art d'occuper, de construire et d'aménager l'espace social avec des choses-dieux; qu'on les appelle idoles ou fétiches peu importe, pourvu que leur façon, leur disposition, leur évolution donnent forme, donc sens, à la matière et à son étendue. À travers la mise en scène, Cendrars et Léger firent se rejoindre créateur(s) et créatures, assignant aux uns la place et la fonction de médiateurs entre les autres, et en particulier, entre l'homme et la femme. Dans La Création du monde, les déités assistent, un peu pataudes mais bienveillantes, aux «forces magnétiques du désir " qui vont unir l'homme et la femme, eux-mêmes évoluant masqués et finissant par entraîner dans une ronde vertigineuse les animaux, les plantes, les sorciers et les féticheurs. Ici, point de chute ni de péché originel: l'accouplement devient l'«acmé " de cette représentation (Abramovic \& Hergott 2000 : 98) où les éléments ne se séparent pas mais se superposent, s'enchevêtrent, s'emboîtent, s'assemblent telles des pièces

25. À noter que les divinités, ou déités selon l'expression retenue par Cendrars, sont au nombre de trois - allusion peut-être à la trinité qu'on appelle sainte - mais sans qu'un ordre hiérarchique ne soit introduit entre elles; elles agissent de conserve. 
d'un Meccano. C'est d'ailleurs, comme il en est du livret original de l'œuvre de Stravinsky, signé par Nicolas Roerich, un printemps, une renaissance que célèbre l'argument de Cendrars, mais sans qu'il soit besoin de faire appel au sacrifice quelque peu barbare du premier : celui d'une jeune femme vierge, dite l'«Élue ». Ici, il n'y en a pas, et il n'y a pas de sacrifice qui précipiterait le temps du rite, du moins le dramatiserait. L'argument du ballet n'a pas d'intrigue, et la musique qui l'accompagne n'est pas une "musique à programme".

En fait, l'ensemble se donna à voir (et à entendre) comme une espèce de ballet mécanique dont le seul rouage consistait en un jeu - ainsi qu'il peut être dit du fonctionnement d'un engin - de formes, de mouvements, de couleurs, de lumières et de sons. L'archaïsme, la magie, en un mot l'expressionnisme de l'art nègre était laissé de côté au profit d'une "machinerie» moderne, massive, complexe qui accentuait la vision essentiellement plastique et - nous y avons déjà insisté - géométrique et cinétique que Léger sembla retenir de cet art. Aussi cette nouvelle et autre genèse que les auteurs du ballet avaient cherché à représenter prit-elle l'aspect d'une construction du monde, pour reprendre le titre du recueil de Marc Augé (1974), plutôt que la tournure dramatique d'une création du monde proprement dite : l'intérêt que, dans son œuvre et dans sa vie, Léger manifesta pour l'architecture y est probablement pour quelque chose (Fauchereau 1994 : 24-28). Dans cette édification, dans ce jeu de construction que des critiques assimilèrent - cela avait été déjà le cas, le calembour en plus, pour Le Sacre du printemps - à un nouveau "jeu de massacre ", Milhaud ne fut pas en reste, lui le "grand barbare», juif et provençal d'origine, brésilien et négro-américain de cour, tenant " un maillet de drummer entre les dents et brandissant l'écarlate drapeau du communisme des tons " avec, comme poursuit le poète José Bruyr (1957 : 409), « sa musique suave et râpeuse, fleurie et cactiforme, à travers laquelle s'ébattent infusoires à ressorts, myriapodes à roulettes et crocodiles en puzzle cubique».

\section{Le son nègre / leçon d'Afrique}

En dépit d'une structure qu'on peut juger régulière, par conséquent classique (Collaer 1982 : 118) : prélude, fugue, rondo, concertino..., mais dont la durée est assez brève comparée aux ballets traditionnels, en particulier romantiques (entre 16 et $17 \mathrm{mn}$ selon les interprétations, soit moitié moins que Le Sacre du printemps ou Pétrouchka qui constituèrent déjà une rupture), la partition écrite par Darius Milhaud pour La Création du monde comporte elle aussi des innovations tant musicales qu'orchestrales, et présente des écarts, voire des ruptures non seulement avec les compositions et 
instrumentations des musiques de scène de l'époque (les changements de rythmes et de formes sur une mesure à $2 / 2$ surviennent sans transition tout comme se produisent les oscillations et superpositions tonales dans le prélude et les cinq mouvements de la pièce où prédominent bois, cuivres et percussions, et qui sont joués en continu, sans aucune pause entre eux), mais avec la veine originelle du ballet et ce qu'il était censé illustrer.

En effet, l'Afrique "archaïque " - celle des chants, des tambours, des balafons, des trompes ou des rythmes superposés ${ }^{26}$ qu'aurait dû faire entendre la cosmogonie extraite par Cendrars d'un fonds mythologique africain authentique - demeure singulièrement absente de la partition. Sauf à travers le jazz en lequel Milhaud puisa sa principale source d'inspiration bien que celui-là fût, comme il le souligne, une "musique mécanisée et aussi précise qu'une machine" (Milhaud 1982: 103) à cause du «halètement de rythmes, brisés, tordus» (Milhaud 1973: 115) qu'enserre une mesure rigoureuse et strictement répétitive à deux ou quatre temps, qui fait d'autant mieux saillir, tel le chuintement ou le hoquet d'un piston, ses accents syncopés, ses brêves séquences mélodiques, ses inflexions tonales, ses gesticulations sonores ${ }^{27}$. C'était là devancer la fameuse thèse du conflit entre le rythme et la mesure - le «serpentement» de l'un et «la rigidité métrique " de l'autre - que formulera trois ans plus tard André Schaeffner (1988: 32, 36) dans son célèbre essai sur le jazz, et par quoi il définira le swing ; et c'était entrevoir le caractère cyclique du jeu du jazz, son mouvement rotatif qui consiste à enchaîner une série de phrases musicales (variations ou improvisations) à partir de la structure harmonique fixe d'un simple thème de douze ou trente-deux mesures, et qui fera toujours retour (Hodeir 1984 : 73). C'était là aussi reconnaître implicitement ce sur quoi Theodor Adorno (2003 : 74) insistera dans son article dévastateur de 1936 à propos du "fétichisme" du jazz qu'il assimile à une "marchandise ", à savoir que le prétendu archaïsme de celui-ci « est aussi moderne que les "primitifs" qui l'ont produit». Leiris (1996: 1150), quant à lui, l'avait perçu comme une irruption du primitivisme dans la civilisation industrielle américaine, un clash, insistait-il, entre le primitivisme et le machinisme.

26. Ces éléments de la musicologie africaine étaient en partie déjà connus à l'époque, par les relations de voyage, les quelques relevés musicaux qui avaient été publiés, les enregistrements sur cylindre réalisés ou par des instruments qui avaient été collectés et exposés dans les musées d'ethnographie (voir Schaeffner 1968, 1988, 1994).

27. La conférence qu'il donne à la Sorbonne le 22 mai 1924 devant le Groupe d'études philosophiques et scientifiques pour l'examen des idées nouvelles, reprise dans Milhaud (1982: 99-105) et d'abord publiée dans L'Esprit nouveau (juillet 1925, n 25 ), s'intitule précisément «Jazz-band et instruments mécaniques. Les ressources nouvelles de la musique" (voir Martin \& Roueff 2002 : 178-181). 
Cette musique était en réalité bien peu "sauvage», et de l'Afrique ne semblait plus conserver que la couleur de peau de ses principaux créateurs et interprètes. De surcroît, ceux-ci s'étaient emparés d'une lutherie d'origine essentiellement européenne, quand bien même se seraient-ils ingéniés à la manipuler, à la déformer, à littéralement la dénaturer par l'emploi de sourdines diverses dont la fameuse sourdine wa-wa imitant la voix humaine, par l'approche percussive des claviers, le jeu des pistons à micourse, les glissandos au quart de ton des trombones, les pizzicatos des cordes, les couinements et les harmoniques des anches, et par le regroupement des percussions (il allait permettre la seule innovation instrumentale due au jazz, c'est-à-dire l'invention du set de batterie). Bien que Milhaud reconnût avoir composé son orchestre "comme ceux de Harlem" (Milhaud 1973: 125), ces derniers ne lui furent pas complètement étrangers. La comparaison ne tient donc pas sa promesse d'exotisme: les singularités organologiques que l'aura soi-disant primitiviste et nègre de Harlem aurait pu produire et développer étaient en réalité fort limitées, en tout cas elles ne lui apparurent ni prêtes à l'emploi ni propices à l'emprunt. Les orchestres noirs uptown jouaient le plus souvent de et avec cette lutherie européenne. Et la formation que Milhaud conçut pour l'exécution de sa partition reproduit, à deux pupitres près, leur agencement. Aucun instrument exotique, folklorique ou populaire n'entra de fait dans l'orchestration de La Création du monde, pas même un banjo, un xylophone, un kazoo, un harmonica, un accordéon ou une batterie (sur ce plan, Stravinsky avait été plus audacieux en introduisant un cymbalum soliste dans son Ragtime pour onze instruments composé en 1918). Imitation sonore, couleur locale, pittoresque instrumental furent résolument écartés par Milhaud. Il n'y avait donc rien dans sa partition ni dans sa formation qui pût évoquer "le tam-tam d'une effrayante bamboula", comme le constatera, non sans fiel ni dédain pour la représentation du ballet et pour le sujet représenté, un ancien journaliste, antisémite et collaborateur notoire, de Je suis partout (Rebatet 1969: 571).

Sur ce plan strictement instrumental, Milhaud s'est inspiré effectivement des ensembles qui accompagnaient les revues et opérettes noires de la Harlem Renaissance, et non pas de ceux, d'un effectif habituellement plus réduit (pour la plupart des sextettes), qui, dès 1917, popularisèrent le jazz de style Nouvelle-Orléans ou Dixieland. Lors de son premier séjour à New York pendant l'automne-hiver 1922, il a probablement assisté à quelques représentations de ces comédies musicales, et notamment à deux d'entre elles: Shuffle Along montée en 1921 à Broadway, sur une musique 28. À qui l'on doit, par ailleurs, l'écriture de deux grands standards du jazz, Sweet Georgia Brown et Them There Eyes. 
du pianiste de ragtime Eubie Blake et des paroles de Noble Sissle, laquelle connut un succès retentissant (504 représentations), ou encore Liza écrite et créée au tout début de 1922 par Maceo Pinkard ${ }^{28}$, dont l'instrumentation se rapproche le plus de celle de La Création du monde (Milhaud 1982: 104 ; Collaer 1982: 118). Ce type de configuration orchestrale où étaient insérés des pupitres de cordes s'accordait assez bien avec ses intentions - «utiliser le style jazz sans réserve en le mêlant à un sentiment classique" (Milhaud 1973: 125) - et avec son esthétique polytonale dans la mesure où les lignes mélodiques pouvaient être décuplées et superposées selon des schémas harmoniques différents, les médiantes majeure et mineure juxtaposées ou alternées, les registres et les timbres séparés puis mélangés, " un seul instrument de chaque espèce amenant la différenciation la plus complète» (Collaer 1982: 89) et prenant ainsi "une importance linéaire nouvelle» (Schaeffner 1988: 110). Ce n'est donc pas la déformation des timbres que recherche Milhaud (sourdines des cuivres, growls, grincements ou hoquettement des anches, par exemple) - en ce sens son orchestre ne sonne pas "comme ceux de Harlem»-, mais leur enchevêtrement, effet peut-être de son "graphocentrisme » qui, plus tard, lui sera reproché par des critiques et musicologues du jazz (Hodeir 1984 ; Schuller 1996).

Formé de dix-sept musiciens solistes ${ }^{29}$, l'orchestre comprend (Milhaud 1929) : un piano, deux trompettes, un trombone, un cor, un saxophone alto, un hautbois, deux clarinettes, un basson, deux flûtes, deux violons, un violoncelle, une contrebasse, un ensemble de percussions (timbales, caisse claire, grosse caisse, tambourins et cymbales), avec priorité de jeu

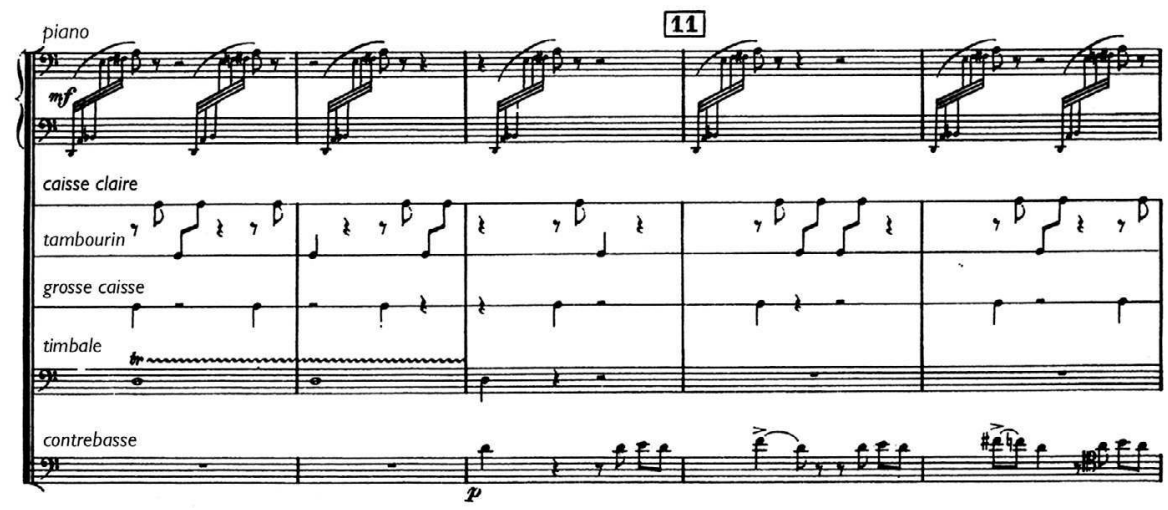

Exposition (62 à la noire) par la contrebasse du sujet de la fugue du ler mouvement de La Création du monde

29. Ce qui tranche là aussi avec les orchestres de ballet traditionnels, d'un effectif généralement plus important, de dimension symphonique. 
donnée au hautbois, au saxophone et aux percussions, sans oublier la contrebasse (instrument soliste plutôt inusité dans la musique savante européenne, tout comme du reste l'était le saxophone) à l'archet de laquelle est confiée, sur fond d'arpèges pianistiques, l'introduction syncopée de la fugue du ${ }^{\text {er }}$ mouvement de La Création du monde: son attaque grave et ronflante sur des notes de blues peut s'entendre comme un pendant (clin d'œil peut-être) au jeu aigu et grinçant - presque injouable sur cet instrument - du basson qui, sur un thème inspiré quant à lui du folklore russe, ouvre Le Sacre du printemps.

Un tel ensemble, note Paul Collaer (1982: 89), était « le plus apte à traduire la polytonalité la plus radicale » vers laquelle Milhaud penchait depuis quelque temps; et c'est peut-être dans cette composition qu'il «exprime le plus entièrement cette sensibilité particulière qui lui faisait entendre la musique comme un rayonnement infiniment varié, venant de tous les points de l'univers à la fois ». Sorte de «musique kaléidoscopique» (ibid. : 77) qui, bien qu'elle fût décalée par rapport à la chorégraphie du ballet, reflétait acoustiquement la mise en scène cubiste conçue par Léger et Cendrars. Elle ne doit cependant pas masquer que c'est l'essence du jazz, sa pulsation orginelle, son idiome musical, c'est-à-dire la carrure rythmique, les altérations tonales, le cisaillement des séquences mélodiques que Milhaud cherchait prioritairement à saisir, à s'approprier puis à transposer dans une partition savante, considérant que ces traits venaient du «fond des âges » et "prenaient racine dans les éléments les plus obscurs de l'âme noire, les vestiges africains, sans doute» (Milhaud 1973 : 115). Pour lui, il ne fait aucun doute que, dans le jazz "nègre », les éléments ethniques ont été conservés, sont demeurés intacts ${ }^{30}$ :

"Le côté primitif africain ", soulignait-il (Milhaud 1982 : 103-104), « est resté profondément ancré chez les Noirs des États-Unis, et c'est là qu'il faut voir la source de cette puissance rythmique formidable, ainsi que celle de ces mélodies si expressives, qui sont douées du lyrisme que seules les races opprimées peuvent produire. Les premiers morceaux de musique nègre publiés sont les negro spirituals, chants religieux d'origine populaire très ancienne [...]. Ces chants ne sont pas d'un sentiment très différent de celui des mélodies qui se retrouvent dans les "blues" dont la forme est l'œuvre de M. [William Cristopher] Handy. Écoutez le Saint Louis Blues, le Aunt Hagar's Children's Blues. C'est la même tendresse, la même tristesse, la même foi que celles qui animaient les esclaves qui, dans leurs chants, comparaient leur sort à celui des Juifs captifs en Égypte et qui appelaient de toute leur âme un Moïse qui les sauverait (Go Down Moses!)».

De sorte qu'on pourrait avancer, paraphrasant James Clifford (1996: 198), que c'est paradoxalement par l'avenir - par le machinisme des Blancs autant d'ailleurs que par le prophétisme des Noirs, dont les negro

30. Quelques années plus tard, ce sera une conception analogue que formulera le critique de jazz Hugues Panassié; voir Ludovic Tournès (1999 : 77 sq.). 
spirituals furent une des expressions privilégiées -, autrement dit en passant par l'Amérique, que l'Afrique "sauvage " serait arrivée et aurait été entendue à Paris, au théâtre des Champs-Élysées... Et c'est bien vers cette Afrique transplantée que Milhaud se tourne en premier lieu, comme si, du fait de cette transplantation qui résultait d'une déportation brutale de nombre de ses habitants, les éléments primordiaux de l'expression musicale africaine devaient apparaître plus nets, plus purs, plus originels, car réduits, ainsi qu'on peut le dire d'une fracture. Tout ce qui restait, en somme, d'une mémoire sociale et culturelle, et qui, sous le joug de l'esclavage, ne pouvait se manifester que dans et par le corps: par des gestes (doigtés percussifs, acrobaties sonores, pas de danse claqués ou chaloupés) ou à travers des voix (psalmodies granuleuses, inflexions gutturales, "appels enchifrenés» comme Roland-Manuel [2000 : 137] qualifia avec une belle justesse de mot l'aspect responsorial des negro spirituals et du jazz). Le chant, la danse, le rythme, en un mot: le musiquer aurait été la seule chose que l'Amérique blanche ne put ôter à ceux qu'elle avait dépossédé de leur propre parler, de leur humanité même, mais grâce auquel ils auraient reproduit et transmis une part de leur africanité, que celle-ci fût jugée atemporelle, authentique, voire de nature biologique comme bon nombre des contemporains de Milhaud le crurent (Cendrars le premier), ou déjà transformée comme lui-même devait le constater au terme d'une expérience qu'on pourrait qualifier de terrain. Car, c'est à des formes composites, à une sorte de "syncrétisme musical» (Schaeffner 1994 : 353) que Milhaud eut en réalité affaire, la simplicité des phrases mélodique et la circularité des figures rythmiques qu’il décela dans le jazz n'étant pas forcément signes de primitivisme, même si elles lui apparurent comme des signes de négrisme, dès lors perçu comme une altération des sons et des tons par rapport au tempérament occidental. L'Afrique qu'il eut donc en tête et coucha sur sa partition n'était pas si archaïque que cela.

De tous les compositeurs européens de musique savante, de tous les musicologues et critiques musicaux qui s'intéressèrent très tôt au jazz, Milhaud est en effet celui qui en eut la connaissance la plus intime, la plus directe et la plus passionnée ${ }^{31}$. Peut-être cette passion fut-elle amplifiée, comme le suggère Jeremy Drake (in Milhaud 1982: 31), par une sorte de sympathie

31. C'est au cours d'un séjour à Londres, en 1920, où il se rendit à l'Hammersmith Palais - salle de bal permanente - que Milhaud eut son premier contact avec le jazz, mais au travers de l'orchestre blanc du pianiste Billy Arnold (qui devait se produire également en France en 1921) : "Il me vînt à l'idée ", note-t-il dans ses mémoires (1973: 100), "d'utiliser ces rythmes et ces timbres dans une musique de chambre, mais il fallait auparavant pénétrer plus profondément les arcanes de cette nouvelle forme musicale dont la technique m'angoissait encore ». 
naturelle qu'il n'aurait pu qu'éprouver, lui le «Français de Provence et de religion israélite» (Milhaud 1973 : 9), vis-à-vis d'un autre peuple déplacé et persécuté. Toujours est-il que la longue correspondance qu'il entretint avec son ami, le diplomate Henri Hoppenot et sa femme Hélène (Milhaud \& Hoppenot 2005), révèle qu'il se comporta, lors de son séjour à New York en 1922, sinon comme un véritable ethnomusicologue (Milhaud n'est certes pas Bartók! Et son intérêt pour le jazz ne sera en fait, au regard de sa longue carrière musicale et de son œuvre profuse, que de courte durée et d'assez mince influence ${ }^{32}$ ) du moins comme un observateur extrêmement réceptif aux phénomènes musicaux inédits et, dit-il, «inouïs " qu'il découvrait. Il cherchera à les étudier sur place, dépensant un "argent fou " et rôdant, comme il le confesse aux Hoppenot ${ }^{33}$, des nuits entières dans les bistrots et bouges de Harlem, notant et transcrivant sur le vif:

"Il n'est pas rare », observait-il (Milhaud $1982: 105)$ ) « d'entendre une négresse chanter la même mélodie pendant plus d'une heure, mélodie souvent poignante et d'un dessin aussi pur que n'importe quel beau récitatif classique, soutenue par un jazz qui forme un fond de mélodies incessamment renouvelées. Les variations sont telles qu'elles prennent l'ampleur d'une symphonie. [...] Là nous touchons à la source même de cette musique, au côté profondément humain qu'elle est capable d'avoir et qui bouleverse aussi complètement que n'importe quel chef-d'œuvre de la musique universellement reconnu!»

L'effort d'analyse qu'il manifesta à l'égard du jazz « nègre ", et l'emprise que celui-ci eut alors sur lui, sont évidemment repérables au niveau du langage musical stricto sensu de La Création du monde. Il suffit d'écouter la manière dont Milhaud utilise les blue notes (inflexion des III et VII e degrés de la gamme), et qui montre que, mieux que quiconque à l'époque (que ce soient Stravinsky, Honegger, Ravel ou même Gershwin qui ne composa sa fameuse Rhapsody in Blue qu'à la fin de 1924, c'est-à-dire un an après la Première de La Création du monde), il en avait parfaitement compris la place dans le phrasé jazzistique tout autant que le parti mélodique et harmonique qu’il

32. Moins de dix ans plus tard, en 1926, lors d'un nouveau voyage aux États-Unis, Milhaud écrira dans ses mémoires (1973 : 158) que "même à Harlem, le charme était rompu pour moi! Les snobs, les Blancs, amateurs d'exotisme, les touristes de la musique nègre avaient pénétré dans ses plus intimes recoins. C'est pour cela que je me retirai ». Au cours de ses longs séjours à Mills College (Oakland, Californie) où il fut invité à enseigner la composition musicale pendant et après la Seconde Guerre mondiale, Milhaud n'aura pas un seul mot (du moins pas à ma connaissance) pour la révolution du be-bop que déclenchèrent Charlie Parker et Dizzie Gillespie à New York, pas plus que pour le jazz cool des années 1950 qui se développa notamment à Los Angeles, ou le jazz modal de Miles Davis à la fin de ces mêmes années - les recherches harmoniques de l'un et les innovations orchestrales et tonales des autres auraient dû pourtant retenir son attention.

33. "J'ai beaucoup rôdé des nuits entières chez les Nègres ", leur écrit-il le 20 février 1923 (Milhaud \& Hoppenot 2005: 68), «c'est ce qu’il y a de mieux à New York. J'ai découvert des bistrots noirs up town avec des jazz populaires magnifiques où des négresses aux voix cuivrées chantaient et dansaient. J'étais très connu là et au mieux avec tous les Noirs ; [...] J'ai dépensé un argent fou.» 
pouvait tirer de leur ambiguïté tonale (Milhaud est alors hanté - je l'ai mentionné plus haut - par la question de la polytonalité, et de façon analogue aux peintres cubistes vis-à-vis de l'art nègre, et croit trouver dans le jazz une justification de ses propres interrogations esthétiques).

Ce sera notamment l'avis du critique, musicologue et musicien de jazz français André Hodeir. Malgré des réticences très prononcées à l'égard de l'alliage entre jazz et musique savante occidentale, estimant qu'il s'agissait là d'une "métamorphose douteuse ", d'une "vaine et absurde entreprise ", d'une « impossible et inutile transplantation " de l'un dans l'autre, et que les œuvres qui en relevaient ont toutes constitué, à ses yeux, de "graves échecs » ${ }^{34}$, parce qu'aucune pièce de jazz, précise-t-il, " ne préexiste totalement à l'exécution" et que la question de l'interprète y est centrale ${ }^{35}$, Hodeir (1981 : 223-239) proposera une des plus fines analyses de la structure musicale de La Création du monde. Il reconnaît justement à son auteur, tout en critiquant l'ingénuité de ses propos et la raideur classique de son écriture musicale, le mérite d'avoir été l'un des rares musiciens européens à sensiblement approcher la signification véritable de la blue note, c'est-à-dire son instabilité tonale, à utiliser la gamme du blues et à essayer de comprendre sinon restituer la rythmique jazzistique.

Milhaud fut non seulement sensible, comme on l'a vu, au langage et aux techniques instrumentales du jazz, à ses racines africaines, à ses cadres et

34. Pour une étude plus mesurée sur ce rapport entre jazz et musique savante, bien que par endroits imprécise sur le plan historiographique, voir l'article de Carine Perret (2003).

35. En se fondant essentiellement sur la partition de La Création du monde et sur les versions enregistrées qu'il a pu en entendre en 1954, année de la rédaction de son étude, Hodeir a raison de souligner ce point, estimant qu'aucun interprète de formation classique, «si habile, si fin musicien soit-il, ne peut faire illusion" sur sa manière de jouer les rythmes du jazz et de restituer son phrasé et ses timbres : "vibratos exagérés, glissandos intempestifs, syncopes heurtées sont le lot de ces entreprises bâtardes" (Hodeir 1981: 237-238). Toutefois, des interprétations relativement récentes de La Création du monde par des musiciens classiques, familiarisés depuis aux techniques et phrasés du jazz - que ce soit par l'écoute ou par leur formation au sein des conservatoires -, rendent justice à l'intuition, aux intentions et à l'écriture de Milhaud, celle-ci serait-elle lue en l'occurrence entre les lignes. Je pense notamment aux versions de l'Ulster Orchestra dirigé par Yan Pascal Tortelier (1992) et de l'Orchestre national de Lille dirigé par Jean-Claude Casadesus (2003), sans oublier la Suite de concert pour quatuor à cordes et piano réduite par Milhaud lui-même, et superbement interprétée par le quatuor Ted Papavrami et le pianiste Éric Le Sage (1999). Elles présentent par moments de beaux phrasés syncopés, presque swingués (voir discographie). On peut cependant s'étonner qu'un ensemble de jazz n'ait pas interprété La Création du monde, comme ce fut parfois le cas pour des compositions du répertoire dit classique (voir notamment les fabuleux arrangements du Peer Gynt de Grieg ou du Casse-Noisette de Tchaïkowski - tous deux musiques de scène - par l'orchestre de Duke Ellington, ou encore l'interprétation, par Gil Evans et Miles Davis, du Concerto de Aranjuez de Rodrigo). Est-ce l'alliage disons a priori que proposait Milhaud entre musique savante et jazz, lequel aurait donc amené à une inutile "surjazzification ", qui aurait mis son œuvre à l'écart? Ou bien, tout simplement une méconnaissance de l'œuvre, même si elle fut reprise en 1939 par le Ballet Theater de New York, avec des danseurs noirs, sous le titre de Black Ritual? 
déterminants sociologiques (expression donc d'un peuple opprimé et marginalisé ${ }^{36}$, mais tout aussi attentif à ses lieux de performance (bistrots, dancings, théâtres), à ses conditions d'exécution et à ses modes de reproduction et de diffusion. Lors de son séjour à New York, il s'était acheté un phonographe et procuré tout un lot de disques de la marque Black Swan ${ }^{37}$ - une de ces nouvelles formes de "reproductibilité technique de l'œuvre d'art » auxquelles Walter Benjamin (1983) consacrera, on le sait, des analyses pénétrantes -, et dont Milhaud allait tirer le meilleur parti. Il les écouta «inlassablement " pendant des semaines entières pour composer La Création du monde ${ }^{38}$, au point d'écrire, non sans humour (ou défi), aux Hoppenot le 4 août 1923 (Milhaud \& Hoppenot 2005 : 73), après qu'il eut achevé sa partition: "Ma musique est intermédiaire entre les phonographes de Broadway et La Passion selon saint Matthieu».

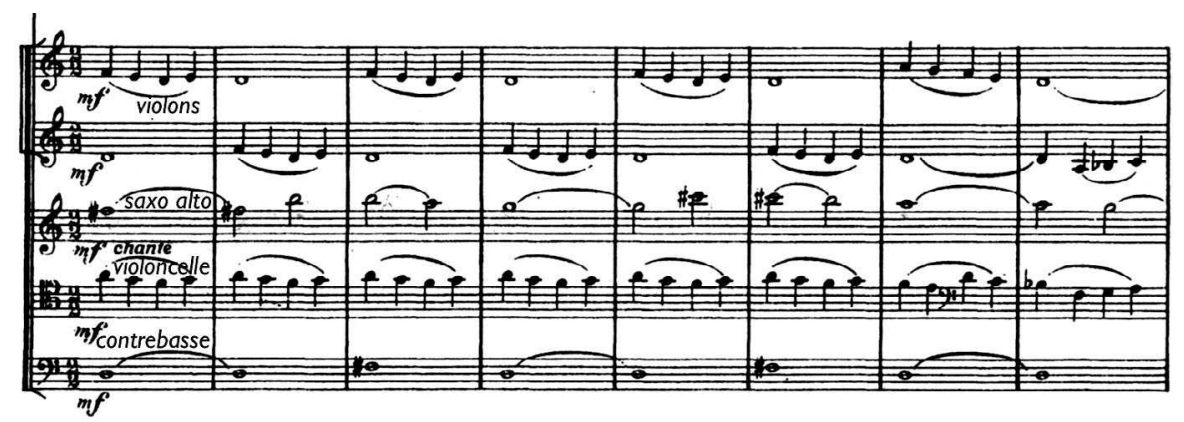

Premières mesures (solo de saxophone, portée 3;54 à la noire) du "Prélude" de La Création du monde

36. Il ira même jusqu’à scandaliser la presse blanche américaine en déclarant dans une interview faite à son arrivée à New York en 1921 que la musique européenne savante avait subi fortement l'influence du jazz, et qu'elle tenait celui-ci en la plus haute estime (Milhaud 1973: 114). Ce qui était exagérément dit et provocant (Milhaud n'était pas à un scandale près!), mais qui le mit brutalement en face de la réalité sociale noire : «Le président du Syndicat [des musiciens négro-américains] m’écrivit une lettre touchante pour me remercier [de cette interview] ; je l'invitai aussitôt à déjeuner sans me douter des complications que cela soulèverait : aucun restaurant ne nous accepta " (Ibid.).

37. Le label Black Swan fondé à New York en 1921 par William C. Handy et Henry Pace - une des premières compagnies phonographiques noires - eut à sa tête, comme directeur artistique, l'hautboïste William Grand Still, et comme directeur des enregistrements, le pianiste et chef d'orchestre Fletcher Henderson qui transposera au niveau d'un grand orchestre (seize à dix-sept musiciens) la polyphonie du jazz Nouvelle-Orléans, fixant à chaque section (cuivres, anches, section rythmique), avec l'aide de son principal arrangeur Don Redman, un rôle bien précis dans l'exécution; il peut être considéré comme le "père" des big bands du jazz. Milhaud l'a sans doute entendu se produire au club Alabama de New York à la fin de 1921, ou, en tout cas, il a joué ses disques une fois rentré à Paris.

38. «En rentrant en France, je jouai inlassablement sur un petit phonographe qui avait la forme d'un Kodak, des disques de la marque Black Swan que j’avais achetés dans une petite boutique à Harlem; plus que jamais, je pensai à utiliser le jazz pour une musique de chambre " (Milhaud 1973: 116). 
Alors qu'on aurait pu s'attendre à ce qu'il citât et se référât, ne serait-ce qu'en raison du titre du ballet, à l'oratorio de Haydn, La Création, c'est celui de Bach qui lui vient immédiatement à l'esprit et qui guidera en partie sa plume: sur une tonalité de ré mineur, le solo de saxophone alto du prélude de La Création du monde (chiffres 1 à 7 de la partition; voir Milhaud 1929, et ci-dessus) évoque sur une mesure à deux temps et de manière presque nonchalante le majestueux prologue choral en $m i$ mineur de l'œuvre de Bach.

La référence à celle-ci peut étonner, et même détonner. Quelles que soient l'inventivité, la complexité, la richesse des timbres et contrepoints de La Passion selon saint Matthieu, la grandeur et la puissance de son orchestration et le cadre liturgique de sa création - elle avait certes de quoi séduire un musicien de la trempe de Milhaud confronté à une "vaste matière ", soit une cosmogonie -, le sujet sur lequel se fonde la composition de Bach n’a évidemment rien de génésiaque, et, ce faisant, ne peut être qu'en porte-à-faux par rapport à l'argument de Cendrars. Dès lors, commencer par une citation ou une paraphrase, fût-elle discrète, rapide, légère si ce n'est désinvolte (le saxophone, à l'époque, ne fait pas partie des instruments concertants nobles), à ce qui était considéré, et l'est toujours, comme un des sommets de la musique savante et religieuse occidentale, mais dont l'instrumentation - ceci n'est sans doute pas un mince détail pour lui - excluait cuivres et timbales, et enchaîner sans transition sur une "petite musique de bastringue " en revanche puissamment cuivrée et syncopée, aux mélismes prononcés, relève soit du contresens soit de la provocation. La grande culture musicale de Milhaud de même que, au propre comme au figuré, sa posture nonconformiste m'amènent à retenir plutôt la seconde hypothèse. D'autant plus qu'il va inverser délibérément le rapport entre spiritualité et matérialité, entre musique religieuse et musique de divertissement, recherchant dans le «machinisme» de l'une (rythmes mécaniques de Harlem, phonographes et phonogrammes de Broadway), et ce jusqu'au fond des sillons ou des arrières mondes noctambulesques new-yorkais (toujours l'obsession de la pulsation originelle, de la survivance, de la trace), une empreinte spirituelle que l'autre, aux proportions orchestrales et chorales monumentales (double chœur mixte, double orchestre à cordes et bois, trois orgues), aux récitatifs et arias grandioses, fortement diversifiés (neuf voix solistes), à la dramaturgie inspirée des écritures expressément dites saintes, était censée restituer et magnifier, mais que Milhaud utilise comme une simple trame, en fait comme élément d'un décor de salle d'attente..., en l'occurrence, et non sans irrévérence, ainsi qu'eût pu le faire et le dire son maître Satie, comme une «musique d'ameublement». 
Dans la fugue qui suit immédiatement le prélude de La Création $d u$ monde ${ }^{39}$, Milhaud entre dans le vif du sujet et reprend presque note pour note le thème d'un blues auquel il fait d'ailleurs allusion dans la citation rapportée plus haut page 428 : le Aunt Hagar's Children's Blues (le plus souvent répertorié sous le titre Aunt Hagar's Blues), composé par William Christopher Handy en 1921 et dont l'orchestre de Fletcher Henderson avait enregistré une version pour le label Black Swan en juin 1922. Il est probable que Milhaud l'a analysée sur disque comme sur partition ${ }^{40}$, autant attentif à ses blue notes, à son dépouillement mélodique ${ }^{41}$, qu'à l'épisode biblique dont s'inspirent les paroles et le titre du blues (voir Annexe II). Selon Handy lui-même, Aunt Hagar's Children's Blues est une «laïcisation" d'un chant choral noir (Porter 2002: 22-23) dans lequel le destin des esclaves africains est assimilé à celui des enfants de "tante » ${ }^{42}$ Hagar, du nom de la servante égyptienne qui, après avoir donné un fils, Ismaël, à Abraham se vit ensuite répudiée, chassée par celui-ci et sa femme Sarah qui, stérile, avait été cependant consentante pour qu'elle partageât la couche de son mari, puis condamnée à errer avec son enfant dans le désert (Genèse XVI ; XXI, 10-21). Pour paraphraser Christian Béthune (2003 : 60),

39. Au chiffre 11 de la partition, mesures 1 à 5 (voir Milhaud 1929, et supra page 427). Avec la romance bluesy du II e mouvement (au chiffre 21 ; Ibid.), cette fugue est sans doute la partie la plus audacieuse, techniquement la plus complexe, mais aussi la plus réussie de la composition, en tout cas la plus analysée et commentée (voir Machlis 1961; Hodeir 1981; Collaer 1982 ; Schuller 1996; Perret 2003).

40. La partition de Aunt Hagar's Children's Blues, avec des paroles de James «Tim " Brymn (après avoir été à la tête de formations militaires noires venues en Europe à la fin de la guerre 1914-1918, celui-ci dirigea notamment l'orchestre de la revue Liza de Maceo Pinkard, voir supra note 28), a été publiée en 1921 par Handy Bros. Music Co. Inc. à New York. Milhaud se l'est sans doute procurée. Robert Goffin (2006: 125) est un des premiers critiques musicaux à avoir signalé la parenté qui existe entre le Aunt Hagar's Children's Blues et le thème de la fugue de La Création du monde. Le musicien et musicologue Gunther Schuller (1997 : 196) est persuadé, quant à lui, tout en insistant sur l'influence qu'eut le Aunt Hargar's Children's Blues de Handy sur la composition de La Création du monde, que c'est la version enregistrée par l'Original Memphis Five en janvier 1923 (et non en 1921 comme il l'écrit) - un quintette de musiciens blancs - que Milhaud aurait rapportée en France en février 1923, et dont il se serait alors inspiré. La mention faite par Milhaud des disques Black Swan m'amène à maintenir plutôt l'hypothèse d'une écoute approfondie de l'enregistrement de l'orchestre de Fletcher Henderson de 1922, d'autant plus que Milhaud détestait ce qu'il appelait le "jazz américain" (i.e. le "jazz blanc») qu'il opposait frontalement au «jazz nègre " (Milhaud 1973: 115, 158 ; 1982 : 104-105). Ce n'est pas, du reste, sans une ironie mordante qu'il évoque un autre orchestre de jazz blanc qui connut une grande célébrité à l'époque, celui de Paul Whiteman, lequel s'était autoproclamé «Roi» du jazz: "une machine bien huilée, élégante ", écrit Milhaud (1973 : 115), "une espèce de "Rolls Royce de la musique de danse" mais dont l'atmosphère restait seulement mondaine et sans imprévu ".

41. Une séquence de cinq notes ( $f a, f a$ ou $f a \#$, do $m i b$, do) qui, transposée, forme le sujet de la fugue de $L a$ Création du monde exposé par la contrebasse (ré, mi, ré, fa \#, ré), repris à la quinte, en réponse, par le saxophone; puis retour au sujet par la trompette. Les trois voix sont ensuite distribuées à l'ensemble des instruments en une progression fortissimo où se déchaînent les percussions.

42. Aunt («tante») est un terme par lequel les Blancs, durant la période esclavagiste, s'adressaient à une Noire d'un certain âge (Levet 2003 : 50). 
on peut avancer que le tragique ne procède pas ici d'une destinée au cours agencé par un seul dieu...

Par cet emprunt manifeste au jazz, au blues, à une exégèse religieuse noire, fût-elle bluesée et donc laïcisée, aussi bien que par l'allusion à $L a$ Passion selon saint Matthieu, Milhaud distord l'idée même de genèse et l'inscrit, comme à contretemps, dans une perspective eschatologique: plutôt que de produire une illustration sonore d'une cosmogonie qui a donc peu à voir avec celle, classique et tragique, de la tradition biblique, il s'agit d'évoquer une régénérescence, en l'occurrence musicale, une re-création, une re-construction du monde, et, en particulier, du monde des sons et des tons. À la suite d'Erik Satie, dont il relève cette boutade (Milhaud 1982 : 143) : «Le jazz nous crie sa douleur et l'on s'en fout ; c'est pourquoi il est beau, réel ", Milhaud considère (Ibid.) que ce phénomène musical découvert par l'Europe à la fin de la Première Guerre mondiale "vînt brusquement bouleverser la musique comme un ouragan qui balayerait les derniers miasmes impressionnistes ", invoquant la "cataracte sonore " à quoi il le compare pour s'en prendre en particulier aux épigones de Claude Debussy, à l'académisme de Paul Dukas, ou même au chromatisme des wagnériens: "c'est sur rythme de valse hésitation », note José Bruyr (1957 : 4002), "que le monde disparu avait glissé dans la catastrophe [i.e. la Première Guerre mondiale]. C'est donc au jazz d'opérer un rythmique rétablissement». Peut-être fut-ce là aussi le rôle que joua l'art nègre vis-à-vis des arts plastiques, et, au-delà, celui de l'Afrique par rapport aux valeurs, représentations et croyances occidentales...

Comme le remarqua pour sa part André Schaeffner (1988 : 109), « le jazz agit d'abord un peu partout comme une secousse dont la musique moderne avait besoin pour voir définitivement clair en elle». Mais voir clair en soi jusques et y compris les mélodies et harmonies que fredonnent nos consciences encore ensommeillées ou trop accoutumées, c'était là une forme de "charité épistémologique " que pouvait nous apporter le passage par l'altérité. La clairvoyance ainsi acquise est cependant à double sens (ce qu'on a longtemps négligé) : elle permet de mieux comprendre ladite altérité. À juste titre, Marc Augé a insité à plusieurs reprises sur ce point, remarquant notamment que l'observation de soi à travers celle des autres n'implique pas une "mythification d'autrui " (Augé 1994 : 83). Dès lors, cette phrase du même Schaeffner, quelque peu provocante d'un point de vue musicologique et sociologique, trouve ici sa pertinence : «Sans la venue préalable du Sacre [du printemps]», écrit-il (1988: 98), «le jazz n'eût aucune chance d'être compris en Europe ». Gageons que sans celle de La Création du monde, la représentation de la Revue nègre en 1925 au même théâtre des Champs-Élysées, où 
flamboyèrent Joséphine Baker et Sidney Bechet (revue du reste financée par Rolf de Maré, le mécène des Ballets suédois), n’eût rencontré le succès, celuici bien populaire, qu'elle eut et qui lui est depuis crédité dans la réception et la diffusion exceptionnelles du jazz en France (Tournès 1999; Brierre 2000 ; Martin \& Roueff 2002 ; Malson 2005).

$\mathrm{Si}$, en fin de compte, persiste chez Milhaud et ses acolytes de $L a$ Création du monde un soupçon de curiosité pour un sentiment religieux, sans doute est-ce du côté d'une forme de totémisme qu'il faudrait le rechercher, un totémisme industriel, machinique, urbain, comme Carl Einstein l'avait finement perçu dans les œuvres de Léger (in Monnoyer 1999: 121), et auquel Milhaud, et surtout Cendrars ne furent pas insensibles. Dans Le Lotissement du ciel (2005b : 269-270), celui-ci ose - malgré la mention, ironique sous sa plume, du Dieu chrétien qui n'est certes pas celui polymorphe et païen qu'il avait cru extraire de l'Afrique noire - un rapprochement que ne désavouerait pas Marc Augé entre les fétiches ou masques nègres et certains éléments de l'architecture urbaine ou industrielle de nos propres sociétés :

«Le totem auquel on s'identifie est l'origine de la chose qui engendre l'être, tout comme aujourd'hui les machines en qui se concrétisent non seulement le génie de l'homme contemporain mais encore tous les rêves de bonheur du genre humain et ses aspirations spirituelles de salvation future, et c'est pourquoi le long des autostrades macadamisées les pompes à essence, par exemple, ressemblent tant aux fétiches des sauvages, mêmes formes stylisées, mêmes couleurs criardes, mêmes ornementations, verre, miroir, cuivre, nickel, ampoules ou perles, sang ou cambouis, même astiquage, les slogans électriques des modernes remplaçant les tatouages sacrés des primitifs, les sigles des compagnies d'essence, les marques des trusts concurrents, les gris-gris de secte des initiés, le même besoin spirituel engendrant la même esthétique pour exprimer la même terreur, Dieu, Dieu le Père, Dieu, et c'est pourquoi les pompes à essence au bord des routes et à la sortie des villes, sans rien dire des hauts fourneaux, des cheminées d'usine et de tout le bataclan industriel, les complexes de tuyauteries, de poutrelles, d'engrenages, de câbles, d'étincelles, de fumées lourdes, de jets de vapeur, de lueurs fulgurantes, de décharges électriques qui font de l'activité de l'homme d'aujourd'hui un spectacle tragique, vont par groupe, font cercle et portent chacun un panonceau comme un masque nègre qui s'anime la nuit et stupéfie. »

Au moins «l'Afrique en tête" dont le ballet $L a$ Création du monde a été une des expressions les plus élaborées de la "crise nègre " des années 1920 (c'est, on l'a vu, l'avis de Michel Leiris; et c'est aussi, musicalement, celui de Gunther Schuller [1992: 220]), a-t-elle permis un élargissement du regard non seulement sur les autres et leurs œuvres mais sur soi et ses 
propres industries, au propre comme au figuré. Jean-Claude Blachère (1981 : 204) a raison de souligner que cette attitude d'esprit devait impliquer un «bouleversement des mentalités blanches» occidentales. Que cela n'ait concerné qu'un milieu d'abord restreint, proprement dit avant-gardiste, n'ôte rien à la puissance de son impact sur le plan des valeurs artistiques et intellectuelles au long cours, notamment au niveau pictural et, surtout, musical.

Qu'on le veuille ou non, et quelles qu'aient été les naïvetés, simplifications, ambiguïtés, voire mystifications auxquelles a donné lieu la recherche d'un primitivisme nègre, il y eut bien là quelques leçons à tirer et à recevoir de l'Afrique : leçons qu'on pourrait qualifier de choses, dans la mesure où elles nous apprirent à voir autrement celles que l'on produisait, qui nous liaient ou, simplement, nous entouraient.

Près de quatre-vingts ans plus tard, dans un ouvrage retraçant son itinéraire intellectuel, Marc Augé (2003b), parle aussi des «leçons de l'Afrique », qui, tournant autour du corps, de la personne, du rite, du symbolique et du sens social, lui permirent d'aiguiser son regard sur le proche et de fonder une anthropologie des mondes contemporains. La démarche, scientifique, n'est pas bien sûr de même type que celle, esthétique, de Cendrars, Léger et Milhaud. Il n'empêche qu'elle s'inscrit dans une continuité je dirais de sensibilité, de réceptivité, de curiosité, d'inventivité - l'anthropologie, par définition, ne peut s'en défaire.

École des hautes études en sciences sociales, Paris Centre d'anthropologie des mondes contemporains, LAHIC, IIAC jamin@ehess.fr

MOTS CLÉSIKEYWORDS : Afrique/Africa - art nègre/african art-anthropologie de la musique/ musical anthropology - jazz - ballet - La Création du mondelBlack Ritual - années folles/roaring twenties -Blaise Cendrars - Fernand Léger - Darius Milhaud. 
ANNEXE I

\section{Argument de la Création du monde *}

Lever du rideau très lent sur la scène noire. On aperçoit au milieu de la scène un tas confus de corps entremêlés: tohu-bohu avant la création. Trois déités géantes évoluent lentement autour.

Ce sont Nzamé, Mébère et Nkwa, les maîtres de la création. Ils tiennent conseil, tournent autour de la masse informe, font des incantations magiques.

La masse centrale s'agite, a des soubresauts. Un arbre pousse petit à petit, grandit encore, se dresse, et quand une de ses graines tombe à terre, un nouvel arbre surgit. Quand une des feuilles de l'arbre touche le sol, elle grandit, se gonfle, oscille, se met à marcher et c'est un animal. Un éléphant qui reste suspendu en l'air, une tortue lente, un crabe, des singes qui glissent au plafond.

La scène s'est éclairée petit à petit pendant la création et à chaque animal nouveau elle s'illumine violemment.

Chaque créature, un danseur ou une danseuse jaillissant du centre, évolue individuellement, fait quelques pas, puis entre doucement dans une ronde qui peu à peu se met en branle autour des trois déités du début.

La ronde s'ouvre, les trois déités font de nouvelles incantations et l'on voit la masse informe bouillonner. Tout s'agite, une jambe monstrueuse apparaît, des dos tressaillent, une tête hirsute se montre, des bras se tendent. Deux bustes se dressent tout à coup, se collent : c'est l'homme, c'est la femme soudainement debout. Ils se reconnaissent; ils se dressent l'un en face de l'autre.

Et pendant que le couple exécute la danse du désir, puis de l'accouplement, ce qui restait par terre d'êtres informes apparaît sournoisement et se mêle à la ronde et l'entraîne frénétiquement, jusqu'au vertige.

Ce sont les Nguils, les imprécateurs mâles et femelles, les sorciers, les féticheurs.

Après une danse effrénée, la ronde se calme, freine et ralentit et vient mourir très calme alentour. La ronde se disperse par petits groupes. Le couple s'isole dans un baiser qui le porte comme une onde.

C'est le printemps.

* Blaise Cendrars, in Anthologie nègre, 2005a : 461-462. 
ANNEXE II

Paroles de Aunt Hagar's Children's Blues, par James Tim Brymn, sur une musique de William C. Handy (192I)*

Old Deacon Splivin, his flock was givin'

The way of livin' right, [... Yes...]

Said he... "No wingin', no ragtime singin' tonight." [...Yeah...]

Up jumped Aunt Hagar, and shouted out with all her might,

[...all her might...].

She said : “Oh... 'tain't no use o' preachin' ,

Oh... 'tain't no use o' teachin';

Each modulation of syncopation

Just tells my feet to dance, and I can't refuse

When I hear the melody they call the blues,

Those ever lovin' blues!"

Just hear Aunt Hagar's children harmonizin' to that old mournful tune!

It's like a choir from on high broke loose! [...Yeah... man...]

If the devil brought it, the good Lord sent it right down to me, [... Yes...]

Let the congregation join while I sing those lovin' Aunt Hagar's Blues !

* Paroles transcrites d'après la version enregistrée par Louis Armstrong en 1954 (voir discographie), et qui, à quelques nuances près (ici signalées en italiques et entre crochets), sont celles de la partition originale. 
BIBLIOGRAPHIE

Abramovic, Norman \& Fabrice Hergott

2000 La Création du monde. Fernand Léger et l'art africain. Paris, Adam Biro.

Adorno, Theodor W.

2003 [1982] Moments musicaux. Genève, Contrechamp. Trad. et éd. de Martin

Keltenecker.

\section{Adotevi, Stanislas Spero}

1998 [1972] Négritude et Négrologues. Paris, Le Castor astral.

Augé, Marc

1977 Pouvoirs de vie, pouvoirs de mort. Introduction à une anthropologie de la répression. Paris, Flammarion.

1979 Symbole, fonction, histoire. Les interrogations de l'anthropologie. Paris, Hachette.

1982 Génie du paganisme. Paris, Gallimard.

1985 La Traversée du Luxembourg. Ethnoroman d'une journée française considérée sous l'angle des mours, de la théorie et du bonheur. Paris, Hachette.

1986 Un ethnologue dans le métro. Paris, Hachette.

1988 Le Dieu objet. Paris, Flammarion.

1992 Non-lieux. Introduction à une anthropologie de la surmodernité. Paris, Le Seuil.

1994a Le Sens des autres. Actualité de l'anthropologie. Paris, Fayard.

1994b Pour une anthropologie des mondes contemporains. Paris, Aubier.

1997 L'Impossible voyage. Le tourisme et ses images. Payot \& Rivages.

2000 Fictions fin de siècle, suivi de Que se passe-t-il? (29 février, 31 mars, 30 avril 2000). Paris, Fayard.

2003a Le Temps en ruines. Paris, Galilée.

2003b Pour quoi vivons-nous? Paris, Fayard.

2006 Le Métier d'anthropologue. Sens et liberté. Paris, Galilée.
Augé, Marc, ed.

1974 La Construction du monde. Religion, représentation, idéologie. Paris, Maspéro.

\section{Barthes, Roland}

1957 Mythologies. Paris, Le Seuil.

Benjamin, Walter

1983 [1936] «L'œuvre d'art à l'ère de sa reproductibilité technique ", in Essais 1935 1940. Trad. de Maurice de Gandillac.

Paris, Denoël-Gonthier : 87-126

Bessis, Raphaël

2004 Dialogue avec Marc Augé. Autour d'une anthropologie de la mondialisation. Paris, L'Harmattan.

\section{Béthune, Christian}

2003 Adorno et le jazz. Analyse d'un déni esthétique. Paris, Klincksiek.

2007 "Minstrelsy", L'Homme 183 : 147162.

\section{Blachère, Jean-Claude}

1981 Le Modèle nègre. Aspects littéraires du mythe primitiviste au XXe siècle chez Apollinaire, Cendrars, Tzara. Dakar, Nouvelles éditions africaines.

\section{Bréchon, Robert}

1971 Le Surréalisme. Paris, Armand Colin.

\section{Breton, André}

1979 [1924] Les Pas perdus. Paris, Gallimard.

Brierre, Jean-Dominique

2000 Le Jazz français, de 1900 à aujourd'hui. Paris, Hors Collections.

\section{Bruyr, José}

1957 Histoire de la musique. Paris, BuchetChastel. 
Caillet, Aude

2001 Charles Koechlin, l'art de la liberté.

Paris, Séguier.

\section{Cendrars, Blaise}

2005a [1921-1923] Anthologie nègre, suivi de Petits Contes pour les enfants des Blancs, Comment les Blancs sont d'anciens Noirs, et de La Création du monde. Éd. de Christine Le Quellec-Cottier. Paris, Denoël.

2005b [1949] Le Lotissement du ciel, suivi de La Banlieue de Paris. Éd. de Claude Leroy. Paris, Denoël.

2006a Du monde entier au cour du monde. Poésies complètes. Éd. de Claude Leroy. Paris, Gallimard.

2006b Blaise Cendrars vous parle..., suivi de Qui êtes-vous?, Le Paysage dans l'ouvre de Léger et de J'ai vu mourir Fernand Léger. Éd. de Claude Leroy. Paris, Denoël.

\section{Clifford, James}

1993 "Négrophilie ", in Denis Hollier, ed., De la littérature française. Paris, Bordas : 844-850.

1996 Malaise dans la culture. L'ethnographie, la littérature et l'art au XX' siècle. Paris, Énsb-a. [Éd. orig. américaine: 1988.]

Collaer, Paul

1982 Darius Milhaud. Genève, Slatkine.

Colleyn, Jean-Paul

2004 "L'alliance, le dieu, l'objet",

L'Homme 170 : 61-78.

Depestre, René

1998 Ainsi parle le fleuve noir. Grigny, Paroles d'aube.

\section{Dozon, Jean-Pierre}

2003 Frères et Sujets. La France et l'Afrique en perspective. Paris, Flammarion.

\section{Einstein, Carl}

1961 [1915] La Sculpture nègre. (Éd. de Jean Laude.) Paris, Médiations.

\section{Goffin, Robert}

2006 [1948] Nouvelle histoire du jazz. Du Congo au bebop. "Introduction" de Carlos de Radzitzky, "Lecture " de Yannick Séité. Loverval, Éd. Labor.

Fauchereau, Serge

1976 Expressionnisme, Dada, Surréalisme et autres ismes. Paris, Denoël.

1994 Fernand Léger. Paris, Albin Michel.

\section{Guiette, Robert}

1994 Monsieur Cendrars n'est jamais là. Éd. de Michel Décaudin. Marseille, Éd. du Limon.

\section{Hodeir, André}

1981 [1954] Hommes et problèmes du jazz. Marseille, Parenthèses.

1984 Jazzistiques. Marseille, Parenthèses.

Jamin, Jean

1982 "Les fantaisies du voyageur", Revue de musicologie 68 (1-2) : 19-35.

Jamin, Jean \& Yannick Séité

2006 "Anthropologie d'un tube des Années folles ", Gradhiva nouv. sér.

4: 5-33.

Kahnweiler, Daniel-Henry

1946 Juan Gris, sa vie, son æuvre, ses écrits. Paris, Gallimard.

1963 Confessions esthétiques. Paris,

Gallimard.

\section{Léger, Fernand}

2004 Fonctions de la peinture. (Éd. de

Sylviane Forestier.) Paris, Gallimard.

Leiris, Michel,

1973 [1939] L'Âge d'homme. Paris,

Gallimard.

1992a Journal 1922-1989. Éd. de Jean Jamin. Paris Gallimard.

1992b Brisées. Paris, Gallimard.

1992c Zébrage. Éd. de Jean Jamin. Paris, Gallimard. 
1996 Miroir de l'Afrique. Éd. de Jean Jamin. Paris, Gallimard ("Quarto»). et al. Paris, Gallimard ("Bibliothèque de la Pléiade").

Levet, Jean-Paul

2003 Talkin' that Talk. Le langage du blues et du jazz. Paris, Kargo.

Lévi-Strauss, Claude

1950 «Introduction" à Sociologie et Anthropologie de Marcel Mauss, Paris, PUF : IX-LII.

1955 "Diogène couché ", Les Temps

Modernes 110 : 1187-1220.

Machlis, Joseph

1961 Introduction to Contemporary Music. New York, Norton.

Malson, Lucien

2005 [1976] Histoire du jazz et de la musique afro-américaine. Paris, Le Seuil.

Martin, Denis-Constant \& Olivier Roueff

2002 La France du jazz. Musique, modernité et identité dans la première moitié du XXe siècle. Marseille, Parenthèses.

Milhaud, Darius

1929 La Création du monde, opus 81a. Paris, Max Eschig (partition pour orchestre).

1973 Ma vie heureuse. Paris, Belfond. [1 ${ }^{\text {re }}$ éd. parue sous le titre Notes sans musique, Paris, Julliard, 1949, revue et augmentée en 1963.]

1982 Notes sur la musique. Essais et chroniques. Éd. de Jeremy Drake. Paris, Flammarion.

Milhaud, Madeleine et Darius \& Hélène et Henri Hoppenot

2005 Conversation. Correspondance 19181974. Éd. de Marie France Mousli. Paris, Gallimard.

Monnoyer, Jean-Maurice

1999 Walter Benjamin, Carl Einstein et les arts primitifs. Pau, Publications de l'université de Pau.

Paulme, Denise \& Jacques Brosse

1956 Parures africaines. Paris, Hachette.

Pastori, Jean-Pierre

2005 La Danse. Des Ballets russes à l'avantgarde. Paris, Gallimard.

Perret, Carine

2003 «L'adoption du jazz par Darius Milhaud et Maurice Ravel. L'esprit plus que la lettre", Revue de musicologie 89 (2) :

311-347.

\section{Porter, Eric}

2002 What is This Thing Called Jazz?

African American Musicians as Artists, Critics and Activists. Berkeley, The University of California Press.

Richardson, John

2007 A Life of Picasso. The Triumphant Years, 1917-1932. New York,

A. A. Knopf.

\section{Roland-Manuel}

2000 [1938] Ravel. Éd. de Jean Roy. Paris, Mémoire du livre.

Sartre, Jean-Paul

1992 Un théatre de situations. Éd. de Michel Contat \& Michel Rybalka. Paris, Gallimard.

\section{Schaeffner, André}

1968 "Musique ", in Georges Balandier \& Jacques Maquet, eds., Dictionnaire des civilisations africaines. Paris, Hazan : 286-289.

1994 [1936] Origine des instruments de musique. Introduction ethnologique à l'histoire des instruments de musique. Paris, Éd. de l'Ehess. 
Schaeffner, André \& André Cœuroy

1988 [1926] Le Jazz. Paris, Jean-Michel

Place.

Schuller, Gunther

1996 Jazz: An Historical Perspective. Duke Ellington, Charles Mingus. The Tanner Lectures on Human Values, Cambridge University. [www. tannerlectures.utah.edu/].

1997 L'Histoire du jazz. Le premier jazz, des origines à 1930. Marseille-Paris, ParenthèsesPUF. [Éd. orig. américaine : 1968.]

\section{Somé, Roger}

1998 Art africain et esthétique occidentale. La statuaire lobi et dagara au Burkina Faso. Paris, L'Harmattan.

\section{Tournès, Ludovic}

1999 New Orleans sur Seine. Histoire du jazz en France. Paris, Fayard.

\section{Tzara, Tristan}

1975 [1917] «Note sur l'art nègre», in Euvres complètes, T. 1, 1912-1924. Éd. d'Henri Béhar. Paris, Flammarion : 394-395.

1981 Grains et issues. Éd. d'Henri Béhar. Paris, Garnier-Flammarion.

\section{Valéry, Paul}

2005 [1919] "La crise de l'esprit ", in Variété I et II. Paris, Gallimard : 13-51.

\section{Williams, Patrick}

1989 " "Les nomades de la porte" : Gitans dans les Rhapsodies gitanes", Cahiers de sémiotique textuelle 15 : 65-85.

2006 "Standards et standardisation. Sur un aspect du répertoire des musiciens de jazz ", L'Homme 177-178 : 7-48.

\section{DISCOGRAPHIE}

Armstrong, Louis

1997 [1954] Aunt Hagar's Blues, in LouIs Armstrong Plays W. C. Handy, New York, Columbia, CD: CK 064925.

\section{Henderson, Fletcher}

1922 Aunt Hagar's Children's Blues, New York, $78 \mathrm{t} / \mathrm{mn}$, Black Swan, matrice $n^{\circ} 2034-A$.

\section{Original Memphis Five}

1923 Aunt Hagar's Blues, 78 t/mn, Pathé, New York.

Milhaud, Darius

1956 La Création du monde, Le Bouf sur le toit, Orchestre du Théâtre des ChampsÉlysées, sour la dir. de Darius Milhaud. Éd. André Charlin, CD: SLC 17.

1992 La Création du monde, Le Bæuf sur le toit..., Ulster Orchestra, sous la dir. de Yan Pascal Tortelier. Chandos Records, CD: Chan 9023.

1999 La Création du monde, Suite de concert pour piano et quatuor à cordes (opus $81 b$ ) réduite par Darius Milhaud lui-même, quatuor Ted Papavrami, avec au piano Éric Le Sage, BMG: CD 74321801032

2003 La Création du monde, Le Bæuf sur le toit, Suite provençale, L'Homme et son désir. Orchestre national de Lille, sous la dir. de Jean-Claude Casadesus. Naxos, CD: 8.557287.

2006 [1978] La Création du monde, Le Bouf sur le toit, Saudades do Brazil,

Orchestre national de France sous la dir. de Leonard Bernstein. His Master's Voice, EMI, CD: 094634580823.

2007 La Création du monde, Le Bauf sur le toit (couplés avec I Got Rhythm et Rhapsody in Blue de George Gershwin), réduction pour piano à quatre mains par Darius Milhaud, interprétée par Jacqueline \& William Benshimon, ATCRL, CD: 012007/1. 